Ann. Sci, forest., 1969, 26 (1), 3-44.

\title{
LE PIN MARITIME DANS LE SUD-OUEST DE LA FRANCE
}

\author{
TABLES DE PRODUCTION PROVISOIRES
}

\author{
N. DECOURT, B. LEMOINE \\ avec la collaboration technique de A. SARToLou \\ Station de Sylviculture et de Production, \\ Centre national de Recherches forestières, 54 - Nancy \\ Institut national de la Recherche agronomique
}

SOMMAIRE

Cette étude fait partie d'un ensemble plus important, visant à mettre rapidement sur pied des tables de production pour les principales essences forestières françaises.

La méthode utilisée est voisine de celles employées pour les tables déjà parues.

Les tables proposées correspondent à une sylviculture visant à produire du bois plutôt que de la gemme. D'autres traitements pourraient être choisis et mis en table facilement à partir des résultats obtenus.

\section{I. - GÉNÉRALITÉS}

\section{1. - Caractères généraux de la région étudiée}

\subsection{Situation et limites}

Le " triangle » des Landes de Gascogne est une zone comprise entre la côte atlantique, les vallées de l'Adour, de la Garonne et la Gironde. La forêt qui la couvre, grande de 1000000 ha, est relativement peu interrompue par les cultures. Elle intéresse trois départements : la plus grande partie des départements des Landes et de la Gironde, une partie du département du Lot-et-Garonne.

La forêt qui nous intéresse présentement, est assise sur la formation qu'il est convenu d'appeler "sable des Landes»). Les formations marginales (graves, sables du Bazadais) sur lesquelles on peut encore rencontrer la forêt en imbrication avec d'autres cultures (telle que la vigne par exemple), n'ont pas fait l'objet d'études de notre part. 


\subsection{Climat}

Nous pouvons le qualifier d'océanique. Il est cependant varié, surtout en ce qui concerne les pluviosités. En effet :

- la pluviosité annuelle varie de $1100 \mathrm{~mm}$ (région dite du Bayonnais) à $700 \mathrm{~mm}$ (Pointe de Grave et Pointe est du Lot-et-Garonne forestier) suivant deux axes sud-nord et ouest-est :

- le régime pluviométrique qui est le rapport de la quantité des pluies de printemps à celle des pluies d'automne (printemps : mars, avril, mai, juin ; automne : septembre, octobre, novembre, décembre) varie de $60 \%$ à $110 \%$ suivant un axe ouestest. Ces deux périodes de pluies sont séparées par un minimum de juillet-août $(60 \mathrm{~mm}$ mensuel environ).

Quant aux températures elles varient suivant un axe ouest-est, mais peu :

- températures moyennes janvier : $6^{\circ}$ à $4,5^{\circ}$

- températures moyennes juillet : $20^{\circ}$ à $21^{\circ}$

En conséquence l'indice moyen d'aridité :

$\mathrm{i}$ moyen $=\frac{\mathrm{i} \text { juillet }+\mathrm{i} \text { année }}{2}$ où $\mathrm{i}$ juillet $=\frac{\text { Pluviosité } \quad \text { juillet } \times 12}{\text { Température juillet }+10}$ et

$\mathrm{i}$ année $=\frac{\text { Pluviosité } \text { année }}{\text { Température année }+10}$ varie suivant les mêmes axes que la pluviosité annuelle et ce de 35 à 25 .

Les températures minimales subissent une diminution brusque quand on passe de la dune littorale à la lande voisine.

La région n'est pas exempte de gelées :

- 15 à 60 jours dans les dunes,

— 47 à 80 jours à Picrroton (près de Bordeaux),

— 67 à 97 jours près de Nerac (Pointe Est du Lot-et-Garonne).

\subsection{Géologie et morphologie}

Un socle tertiaire, constitué en grande partie par une nappe d'épandage, est à l'origine des sables des Landes. Ce socle fut remanié au cours d'une période humide par les rivières qui en trièrent les matériaux :

- les argiles furent drainés vers la mer,

- les sables, repris au cours des périodes xérothermiques par les vents d'Ouest violents transportant également des sables d'origine côtière furent étendus sur toute la surface de la région.

Il en résulte deux types de paysages :

- la bande côtière des dunes : 
- la lande d'altitude maxima $158 \mathrm{~m}$ à l'est, comportant des rides à peine marquées et quelques dunes à proximité des vallées. Dans cette lande on peut trouver deux types hydrographiques : la grande lande, au centre de la région est peu drainée tandis que les petites landes sont plus favorisées de ce point de vue.

\subsection{Les sols}

Il faut distinguer deux unités :

- les dunes ont un sol squelettique avec amorce de différenciation ;

- les landes : le retour des périodes humides a provoqué une podzolisation nuancée suivant le caractère hydromorphe variable d'un endroit à l'autre. On peut en effet rencontrer des sols à plans d'eau situés à des profondeurs variables (certaines stations sont submergées en hiver, d'autres ont un plan d'eau situé sous le sol). On peut rencontrer :

- des sols tourbeux,

- des podzols humiques avec horizon B peu dur,

- des podzols humoferrugineux avec horizon cendreux très distinct et horizon B dur ( ( alios $»)$. II est à noter que l'« alios » peut résulter d'une évolution pédologique relativement récente, ou bien être " fossile ».

Quelles sont ies qualités de ces sols ? Nous les décrirons sous deux aspects :

- la capacité de rétention en eau est souvent faible, argiles et limons fins étant peu abondants ; elle est surtout assurée par la matière organique. La moyenne des pourcentages d'argile ou de matière organique est inférieure à $2 \%$;

- analysés sous le point de vue de la nutrition minérale des végétaux, ils semblent peu favorables à la production. Très peu d'éléments minéraux ( $\mathrm{pH} \mathrm{4,5}$ à 5), le phosphore faisant le plus défaut au Pin maritime ainsi que le prouvent les résultats spectaculaires des expériences de fertilisation.

\section{2. - Le pin maritime dans la région}

Le Pin maritime ( Pinus pinaster AIT.) existe à l'état spontané dans la région, qui fait partie de la zone atlantique de l'aire de cette espèce, l'autre partie de cette aire étant méditerranéenne.

D’une façon générale, le Pin maritime tolère bien les sols pauvres, sableux et acides. Il convient donc à la plupart des sols de la région à condition toutefois qu'ils soient suffisamment bien drainés. Cela explique la grande extension de cette essence dans tout le Sud-Ouest de la France, d'autant plus qu'aucune autre essence connue ne semble aussi bien convenir tant au climat qu'aux sols de cette partie de notre pays.

Ainsi s'explique la naissance à partir de la fin du $18^{\mathrm{e}}$ siècle d'une immence forêt artificielle qui a fait passer les surfaces boisées en Pin maritime de 100000 ha, à cette 
époque, à environ 1000000 ha aujourd'hui. Il n'est pas nécessaire de s'étendre ici sur la relative fragilité d'une telle forết sensible aux incendies (300 000 ha détruits de 1938 à 1949), aux attaques de nombreux parasites et au froid, tout au moins pour certaines provenances (Pins du Portugal).

Cette forêt est par ailleurs en pleine transformation. Les reboisements de plus en plus rationalisés, s'appuient sur les progrès des sciences forestières. Les semis en bandes sur sol travaillé et fertilisé sont passés dans la pratique courante. L'usage de semences de qualité - et bientôt de graines sélectionnées - se répand également. Enfin, I'utilisation du bois pour la papeterie et le sciage prend de plus en plus le pas sur celle de la gemme. Nous avons tenu compte de cette évolution dans notre étude.

\section{3. - Documentation et études antérieures}

Nous ne possédons que fort peu d'études sur le plan de la Sylviculture et de la Production proprement dites, c'est-à-dire, traitant des peuplements de Pin maritime non sélectionné génétiquement, et croissant sur des Stations non améliorées par la fertilisation.

J. Guinaudeau et J. Parde (1963) utilisent les données des placettes permanentes de dunes et de quelques placettes temporaires, de dunes elles aussi. Cette étude donne « la mesure $»$ de certaines modalités de croissance du Pin maritime dans la région. Nous commenterons ces résultats utilisés pour la construction de table de production, en lieux et places dans la présente publication.

P. Lallemand, J.P. Leonard, J. Martin, J.P. Mauge et R. Navarre (1960 et 1966) donnent un tableau complet des aspects de la culture du Pin maritime dans la lande. Une étude prospective sur la croissance des peuplements de pins, fertilisés ou non, complète ce tableau.

B. Lemoine (1967) présente les résultats du dispositif expérimental le plus ancien de lande. L'âge de cette plantation (16 ans) limite l'intérêt que ce dispositif aurait pu avoir en tant que dispositif expérimental de lande pour une utilisation éventuelle en vue de la construction de la table de production.

L'Inventaire Forestier National a publié ses résultats relatifs aux trois départements landais. Nous ne pouvons faire mieux ici que de souligner l'importance que revêt particulièrement l'évaluation des ressources en bois dans cette région de sylviculture intensive.

A. OudiN (1938) a publié une étude très détaillée sur une production qui ne nous occupe pas ici : la résine (ou gemme).

En dehors de ce type d'étude portant strictement sur l'aspect " Sylviculture et Production $n$ du Pin maritime, il en existe d'autres qui ne relèvent pas de notre discipline mais dont il faut dire ne serait-ce que quelques mots car les résultats des travaux en question ont et vont avoir de grosses répercussions. II s'agit du reste de "Production ”) au sens large. Chronologiquement, nous distinguons d'abord la publication de J. Guinaudeau, G. Illy, J.P. Mauge et F. Dumas (1963) relative à l'essai de fertilisation minérale de Mimizan. Maintenant, il n'y a plus guère de repeuplements qui 
ne soient exécutés sans travail du sol ni fertilisation. Enfin, G. ILLy (1967) conclut quant aux résultats de plusieurs années d'efforts qu'il a consacrées à l'amélioration génétique du Pin maritime. Les succès de la sélection individuelle vont pouvoir être bientôt exploités sur le plan pratique.

L'équipe du Laboratoire de Biologie végétale de la Faculté des Sciences de Bordeaux, étudie, sous la Direction du Professeur DAvid, le Pin maritime sous un angle plus proprement physiologique. Une meilleure connaissance du déterminisme de la croissance devrait nous permettre de mieux résoudre les problèmes relatifs à l'amélioration de la production.

\section{4. - Placettes permanentes et placettes semi-permanentes}

\subsection{Placettes permanentes}

Des places d'expérience du type ancien bien connu en France, furent installées il y a une quarantaine d'années dans deux forêts domaniales des dunes, distantes d'une centaine de kilomètres suivant un axe nord-sud. Ces dispositifs, dits de Hourtin et de Ste-Eulalie, du type factoriel mais hélas sans répétitions étaient destinés à étudier la production de résine (gemme) suivant le type de traitement sylvicole et l'action sur la production en bois de différents types d'éclaircies et du gemmage.

Malheureusement, ce dispositif expérimental intéresse uniquement la dune et en ce qui concerne celle-ci, une scule classe de productivité que l'on peut qualifier de moyenne, elle-même située fort en-dessous de la classe de productivité moyenne des landes. Les hauteurs dominantes "plafonnent " en effet à $17 \mathrm{~m}$ à l'âge de 50 ans alors que l'on rencontre dans le Bayonnais des hauteurs à maturité pouvant atteindre $27 \mathrm{~m}$... voire près de $30 \mathrm{~m}$, pour des stations privilégiées.

Cependant, ces placettes, hormis toutes considérations de sylviculture, nous ont éclairés sur bien des points, en particulier :

- I'exploitabilité matière est d'environ 40 ans, ce qui situe Pinus pinaster comme une essence à révolution courte par rapport aux autres essences résineuses,

- étant donné que ces placettes ont été fort bien suivies et exemptes de catastrophes, que la production depuis l'origine est bien connue, on a pu construire la loi de Eichhorn valable pour les dunes (Production totale bois-fort $=\mathrm{f}(\mathrm{Hg} \mathrm{ou} \mathrm{H} ø)$. Pour des raisons qui seront exposées plus loin, cette loi n'est pas transposable aux peuplements de landes.

Le résumé des résultats obtenus figure aux tableaux 1 et 1 bis qu'on a fait débuter à 33 ans, au début de la période de gemmage par manque de place. Voir aussi la figure 2 .

\subsection{Le dispositif semi-permanent}

De ce qui vient d'être dit ci-dessus et dans les généralités relatives au milieu naturel, découlait la nécessité d'une implantation d'un dispositif complémentaire de placettes semi-permanentes assez important. 


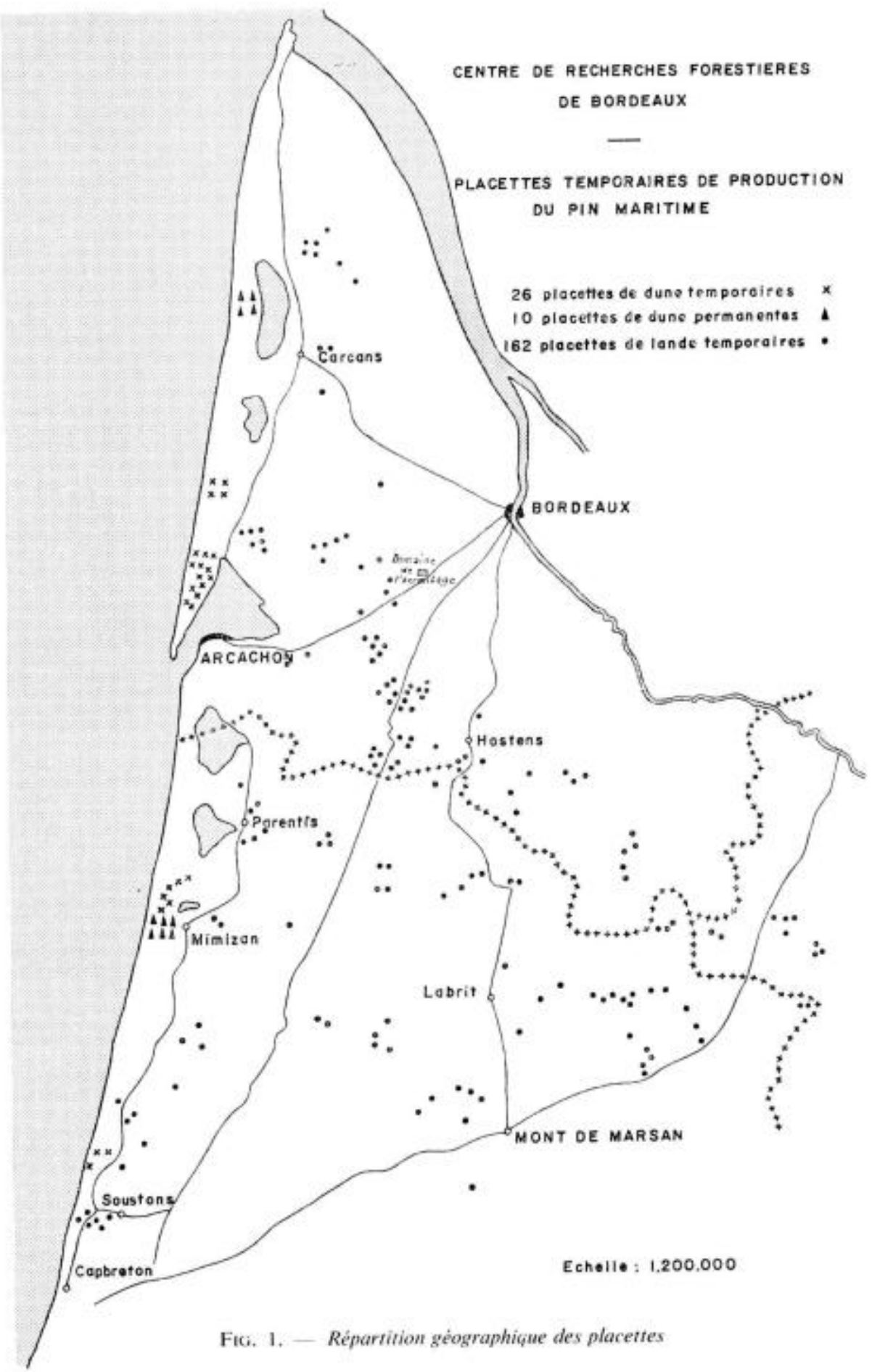


Ainsi que le montre la figure 1, des placettes ont été installées dans les dunes du nord au sud (26 placettes seulement car les dunes bénéficient d'un dispositif permanent valable) et dans les landes (162 placettes). En ce qui concerne cette dernière unité, elles ont été réparties dans toute la région. De plus, dans une zone donnée, il a été choisi des placettes sur stations aussi variées que possible. En particulier, le système de classification des stations relatif au pédoclimat, s'est appuyé sur la végétation. Suivant une humidité décroissante, on rencontre en effet : molinie, ajonc nain, fougère aigle, callune et bruyère cendrée, enfin hélianthène.

Certaines des placəttes ont été remesurées 4 ans après la première mesure, quelques-unes qui sont du reste des placettes de dunes, ont été remesurées deux fois. Nous avons pu utiliser ainsi 17 " doubles mesures $»$ pour les dunes et 21 " doubles mesures $n$ pour les landes.

Nous ne reviendrons que fort brièvement sur la technique d'implantatipn de ces placettes, par ailleurs décrite dans les publications antérieures (DECOURT, 1965, page 263 et 1967, page 53). Rappzlons qu'une placette de production doit être homogène sur toute sa surface du point de vue des hauteurs, des âges, de la densité et sur le plan phytoscciologique.

\section{II. - MÉTHODES}

\section{1. - Problèmes liés à la pratique du gemmage}

Le gemmage entraine deux types de difficultés. L'une fondamentale, concernant la perte de production liée à cette pratique et posant le problème de la construction de deux types de tables différentes pour peuplements gemmés d'une part, pour peuplements non gemmés d'autre part ; lautre de nature méthodologique, les cares de gemmage rendant les mesures de circonférence imprécises,

\subsection{Gemmage et production}

Le gemmage provoque-t-il une perte de production ?

II s'agit bien sûr de la pratique du gemmage à vie, celle du gemmage à mort des places venant en exploitation ne posant pas de probleme en ce qui nous concerne.

Dés 1938, Oudin remarque une diminution sensible de l'accroissement en circonférence des arbres gemmés. Ses observations portent sur une période de 4 ans. II en conclut que le gemmage peut amener une baisse de la production en bois, par arbre, de l'ordre de 10 à $15 \%$.

Davio (1961) ne décèle pas de perte de production à partir d'une série d'analyses de tiges. Par contre, ce même auteur, dans une étude récente (1967) portant sur 75 arbres répartis en 3 lots. conclut à une perte de l'ordre de $15 \%$ pour le gemmage activé à l'acide sulfurique et de l'ordre de $23 \%$ pour le gemmage au hapchott.

On ne retrouve pas cet effet au niveau des peuplements. GuINAUdEAU et PARDE (1963) chiffrent la perte à $5 \%$ de la production totale. Les résultats tirés de nos placettes permanentes de Ste-Eulalie et de Hourtin, suivies pendant 40 ans et gemmées depuis 20 ans permettent de comparer l'effet de l'intensité des éclaircies et du gemmage sur la production de bois des peuplements. La perte due au seul gemmage, n'est pas apparente. Par contre, la pratique des éclaircies fortes - traditionnellement liée au gemmage dans la région - entraine manifestement une perte de production importante.

La figure 2, illustre ces constatations. A hauteur égale, peuplements gemmés et non gemmés. ont des productions voisines si l'intensité des éclaircies est la même. Par contre, la production des parcelles fortement éclaircies, est très sensiblement inférieure. Ajoutons qu'aucune diminution de la hauteur moyenne n'est liée au gemmage. 
PIN MARITIME (DUNES)
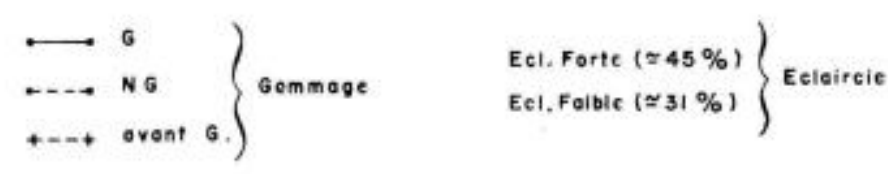

$4004_{-}^{\text {It } \mathrm{m}^{3 / h a}}$

300 -

200
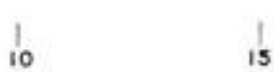

$\underset{20}{1} \rightarrow H G(m)$

Fic. 2. - Production totale-Gemnage et intensité d'éclaircie 
II n'est pas exclu que le ralentissement de croissance constaté chez les individus gemmés, soit en partie compensé par une croissance légèrement accrue de leurs voisins non-gemmés, la concurrence jouant pour l'ensemble du peuplement un rôle compensateur.

En conclusion, notre table de production, envisagée pour des peuplements non gemmés, semble pouvoir donner des renseignements utiles pour des peuplements gemmés. Par ailleurs, un certain nombre de données tirées des placettes gemmées, pourront être utilisables à l'exclusion bien sûr de celles concernant la circonférence à $1,30 \mathrm{~m}$.

\subsection{Difficultés dendrométriques}

Les cares de gemmage remontant jusqu'à 3 et $4 \mathrm{~m}$ de hauteur, la mesure de la circonférence à $1,30 \mathrm{~m}$ n'est plus possible. Les placettes gemmées ont done fait l'objet de cubage établi ả partir de mesures de circonférences faites à $3,0 \mathrm{~m}$ ou à $4,0 \mathrm{~m}$ selon les cas. Par ailleurs, ont été établies des relations mathématiques permettant de passer de la circonférence à $3,0 \mathrm{~m}$ ou à $4,0 \mathrm{~m}$ à la circonférence moyenne qu'aurait à $1,30 \mathrm{~m}$ un peuplement de même hauteur et de même volume (annexe 4).

\subsection{Gènéralités}

\section{2. - Méthodes de calcul}

Les méthodes utilisées sont les mèmes que celles déjã employées pour les tables précédentes ; Pin sylvestre, Pin Laricio de Corse en Sologne (Decourt, 1965); Douglas dans le nord-est du Massif Central (DecourT, 1967). On pourra aisément s'y reporter. Elles comportent l'établissement d'un certain nombre d'équations fondamentales, et leur application à un modèle mathématique reflétant ce qui se passe dans un peuplement soumis à une sylviculture donnée. L'originalité de la présente étude provient du fait que l'on disposait de doubles mesures assez nombreuses, et que, de plus, il nous a fallu distinguer entre peuplements de dunes, et peuplements de landes.

\subsection{Calcul des équations fondamentales}

Les équations fondamentales, à l'exception de celles reliant la hauteur à l'âge, sur laquelle nous reviendrons, ont été calculées par la méthode, maintenant classique, des régressions progressives (Tomassone, 1967 ; MarTIN, 1965).

Les résultats résumés dans le tableau 2, font apparaître que, pour le Pin maritime comme pour les autres essences, on obtient une très bonne "explication $*$ des variables dépendantes ( $\left.{ }^{1}\right)$, sans que l'âge des peuplements intervienne de façon significative.

On arrive ainsi à expliquer la circonférence moyenne $C_{g}$. à partir de la hauteur moyenne $H_{\sigma}$. et du nombre de tiges à l'hectare $N$; le volume sur pied $V$, à partir de la surface terrière $G$ et de la hauteur moyenne ; la circonférence dominante $C_{0}$ à partir de $C_{g}$ et de $N$. La hauteur dominante $H_{0}$ est elle-même étroitement liée à la hauteur moyenne par une relation linéaire.

Enfin, le rapport $\lambda=\frac{V_{w}}{V_{g}}$, qui définit la nature de l'ćclaircie par le rapport du volume moyen de l'arbre enlevé $v_{m,}$, au volume de l'arbre moyen du peuplement avant éclaircie $V_{p}$, est lié de façon satisfaisante au nombre de tiges sur pied, c'est-à-dire, à l'intensité de l'éclaircie (cf. plus loin 3.1.).

Le tableau 2 montre de plus qu'il existe une différence sensible entre les coefficients des équations pour les peuplements de landes et pour ceux de danes. L'étude statistique a établi que ces différences sont significatives. Le choix entre un modèle unique (landes + dunes) et un modèle à deux régressions, a été réalisé en comparant la part de variabilité expliquée par chaque modẻle selon la méthode de comparaison des régressions exposée au Séminaire de Biométrie de I'INRA en mars 1968 (Tomassone et Milufr, 1968).

Les équations faisant intervenir la circonférence ou la surface terrière, ont, bien sûr, été calculées sans utiliser les placettes gemmées.

On remarquera la bonne précision des ajustements obtenus d'après les valeurs des écarts-types résiduels $(\sigma)$ et des coefficients de corrélations multiples $R$. Le volume sur pied, en particulier, peut ètre estimé à partir de la surface terrière $G$, et de la hauteur moyenne $H_{g}$ avec une erreur - type de l'ordre de $5 \%$ 


\section{TABLEAU 2}

Equations fordamentales

(Voir les explications dans le texte)

$$
\text { Duns } N=42 \quad \text { LANDFS } N=104
$$

1 e'

$$
C_{g}=a+b H_{g}+c / N
$$

$\begin{array}{cc}a & 3,6056 \\ b & 4,0712 \\ c & 7102,6591 \\ \sigma & 4,58 \\ R & 0,982\end{array}$

$\begin{array}{lr}a & -0,1529 \\ b & 0,8509 \\ c & 0,9450 \\ \sigma & 0,050 \\ R & 0,992\end{array}$

$$
\begin{array}{r}
-0,5864 \\
0,9186 \\
0.9973 \\
0,046 \\
0,996
\end{array}
$$

$3^{\circ}$

$$
H_{0}=a+b H_{9}
$$

$\begin{array}{ll}a & 1,1256 \\ b & 0,9903 \\ \sigma & 0,334 \\ R & 0,995\end{array}$

$$
C_{0}=a+b C_{s}+c / N
$$

$\begin{array}{lr}a & 14,6141 \\ b & 1,0963 \\ c & -3293,7705 \\ a & 2,200 \\ R & 0,995\end{array}$

0,993

$$
\begin{aligned}
5^{\circ} \frac{v m}{V_{v}} & =91,30-0,0342 N+0,0426 N^{2} \cdot 10^{-4} \\
R & =0.845
\end{aligned}
$$

\subsection{Indice de productivité}

Nous avons retenu comme indice de productivité la hauteur du peuplement sur pied, à un âge déterminé. Parmi les hauteurs possibles, nous continuons à employer de préférence les deux suivantes :

- la hauteur moyenne $H_{g}$, hauteur de l'arbre de surface terrière moyenne, étroitement liée, comme on vient de le voir aux caractéristiques volumétriques du peuplement, mais qui subit de fâcheux à-coups techniques lors des éclaircies;

- la hauteur dominante $H_{0,}$, hauteur de l'arbre de surface terrière moyenne des 100 plus gros arbres à l'hectare. Cette hauteur est un bon indice biologique en raison de son invariance lors du passage d'une éclaircie. Ces deux hauteurs sont étroitement liées par une relation linéaire, ce qui nous permet 
d'utiliser l'une ou l'autre dans les calculs statistiques. Nous avons préféré nous servir de la hauteur moyenne dont la détermination est beaucoup plus précise dans nos placettes, la hauteur dominante n'ayant pas toujours été évaluée avec soin dans les plus anciennoes placettes et notamment dans les placettes permanentes. En présence d'un peuplement déterminé, il vaut mieux, bien sûr, utiliser le plus stable des deux paramétres, c'est-à-dire, la hauteur dominante.

\subsection{Courbes de croissance en hauteur - Relation $\mathrm{H}=f(A, I)$}

II s'agit d'ajuster aux données un faisceau de courbes de croissance, correspondant à différentes classes de fertilités d'indice 1. Cet indice est défini ici comme la hauteur moyenne à 40 ans. En fait, nous verrons que nous nous sommes limités à 5 classes de fertilité correspondant aux indices 12,15 , $18,21,24$. Les tables de production donnent la correspondance pour des indices définis à partir de la hauteur dominante.

La méthode utilisée ici est nouvelle. Elle se base sur le fait qu'on possédait des mesures valables de la hauteur moyenne répétées deux fois, et parfois trois, dans certaines placettes, à des intervalles d'âge de 4 ans. La figure 3 montre ces doubles mesures reliées en pointillé. On disposait ainsi, à une petite approximation près, d'un certain nombre de valeurs de la dérivée $\frac{\mathrm{d} H}{\mathrm{~d} A}$, valeurs elles-mêmes fonctions de $H$ et de $A\left({ }^{1}\right)$, soit 21 valeurs pour des peuplements de landes et 38 pour des peuplements de dunes, assez largement réparties dans tout l'éventail des âges et des hauteurs.

L'ajustement de l'équation $\frac{\mathrm{d} H}{\mathrm{~d} A}=f(A, H)$ fournit une équation différentielle du ler ordre, dont l'intégration, lorsqu'elle est possible, permet d'obtenir le faisceau de courbes de croissance en hauteur cherché.

Un grand nombre d'équations ont été essayées. Finalement, pour la hauteur moyenne $H_{p}$, l'équation la meilleure s'est trouvée être, tant pour les landes que pour les dunes de la forme :

$$
\frac{\mathrm{d} H}{\mathrm{~d} A} \frac{l}{H}=a+\frac{b}{A}
$$

De plus, on a ainsi pu vérifier de façon objective que l'équation pour les landes n'était pas significativement différente de l'équation pour les dunes. C'est donc l'équation commune (landes + dunes) qui a été retenue :

$$
\frac{\mathrm{d} H}{\mathrm{~d} A} \frac{I}{H}=-0,02323+\frac{1.5067}{A}(R=0,92 ; N=59)
$$

Le faisceau de courbes de croissance a donc pour équation :

$$
H=K \times A^{1,5067} \times e^{-0,02323} A
$$

(e est la base de logarithmes népériens et $K$ une constante positive arbitraire).

Ces courbes ne sont croíssantes que jusqu’à un âge, $A=b / a$, c'est-à-dire, dans le cas qui nous occupe, jusque vers 65 ans, âge correspondant à peu près à nos placettes de landes les plus âgées. Les branches croissantes seules se trouvent donc dans les limites de nos observations et présentent un intérêt pour nous. Elles passent par l'origine et possèdent un point d'inflexion pour $A=\frac{\sqrt{b}-b}{a}$, c'est-à-dire, pour $A=12$ ans. On peut remarquer en outre que ces courbes rentrent dans la catégorie des $«$ courbes proportionnelles $\cdots$ fréquemment utilisées par les auteurs américains puisque pour deux courbes quelconques $H_{1} / H_{2}=$ Cte quelque soit $A$ (voir Bruce et SCHUMACHER, I950 et également Curris, 1964).

La constante $K$ pourrait jouer le rôle d'un indice de productivité car elle est proportionnelle à $I$.

$$
\begin{aligned}
& K=I \times 40^{-1,5067}+e^{0,02323 \times 40} \\
& K=\mu \times I
\end{aligned}
$$

(1) $H$ est ici la hauteur moyenne et $A$ l'âge du peuplement. 

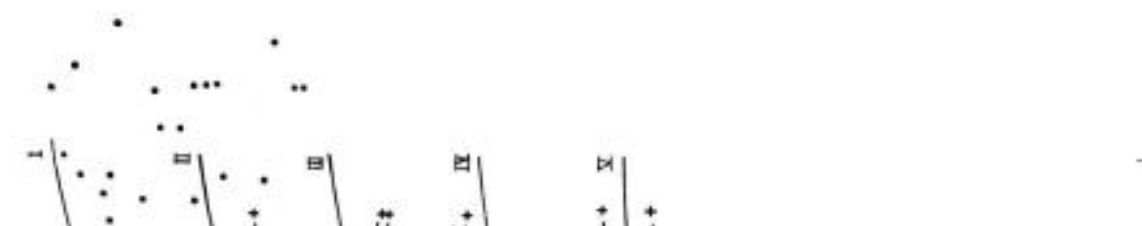

$\begin{array}{ll}: & \vdots \\ \vdots & \vdots \\ 0 & \Xi \\ 1 & a \\ . & +\end{array}$
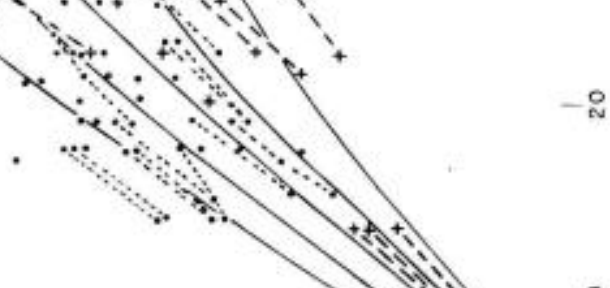

$\bar{E}$
$\dot{0}$

!

$\stackrel{1}{2}$

$\stackrel{1}{0}$

$-10$

Fic. 3. - Croissance en hauteur en fonction de läge et de la classe de productivité 
Pour nos 5 classes de productivité, les valeurs de $K$ sont les suivantes :

$\begin{array}{ccc}\text { CLASSE } & I & K \\ 1 & 24 & 0,234361 \\ 2 & 21 & 0,205066 \\ 3 & 18 & 0,175771 \\ 4 & 15 & 0,146476 \\ 5 & 12 & 0,117180\end{array}$

Ces classes sont les mèmes pour les landes et les dunes à ceci près que la classe I n'existe pas pour les Dunes et que la classe 5 correspond à un niveau de productivité exceptionnellement bas pour les Landes.

La bonne adaptation de ces courbes de croissance au Pin maritime est confirmée par une série de vérifications tout à fait indépendantes de la façon dont elles ont été calculées. On constatera sur la figure 4 , la très bonne concordance du modèle (courbes calculées) avec :

- la courbe moyenne des hauteurs pour les landes et la courbe moyenne pour les dunes; ces courbes auraient servi de "courbes guides" si nous avions appliqué la méthode de Bruce et ScHUMACKER (1950) ;

- la courbe moyenne de croissance en hauteur du dispositif permanent de Ste-Eulalie, qui correspond donc à une courbe réellement observée ;

- la courbe moyenne de 40 analyses de tiges réalisées dans les peuplements de Dunes par le professeur DAviD (1961) de la Faculté des Sciences de Bordeaux.

Notons enfin que le calcul fait à partir des hauteurs dominantes donne des résultats analogues, avec cependant un ajustement moins bon de l'équation différentielle.

\subsection{Conclusion}

On dispose done de toutes les relations nécessaires à la construction de tables de production par la méthode déjả exposée par ailleurs. Ces relations se présentent toutes sous forme d'équations, ce qui a permis d'une part de mettre en évidence de façon objective la nécessité de construire deux séries de tables, une pour les landes, et une pour les dunes et ce qui permettra d'autre part d'automatiser complètement le calcul des tables (cf. 3.2.).

$$
\text { III. - TABLES }
$$

\section{1. - Choix du régime d'éclaircie}

Le visiteur pourra s'étonner, et à bon droit, des densités extrêmement faibles rencontrées dans les peuplements de Pin maritime, par rapport à celles observées dans les peuplements d'autres essences résineuses. A cela deux raisons : la première est que le Pin maritime est une essence de lumière particulièrement exigeante à cet égard ; de plus, tout au moins jusqu'à il y a quelques années, on estimait que pour favoriser la production individuelle de gemme des pins, il fallait pratiquer des éclaircies extrêmement fortes. En fait, en ce qui concerne ce dernier point, il s'est révélé à la lumière des résultats des placettes permanentes que le rendement en gemme à l'ha n'était que très peu augmenté par la pratique d'une éclaircie forte. Ajoutons à cela que le marché de la gemme subit une grosse dépression, qui semble irréversible, et nous comprendrons mieux que les sylviculteurs s'orientent maintenant vers une sylviculture plus raisonnable car mieux adaptée à la production du bois. 


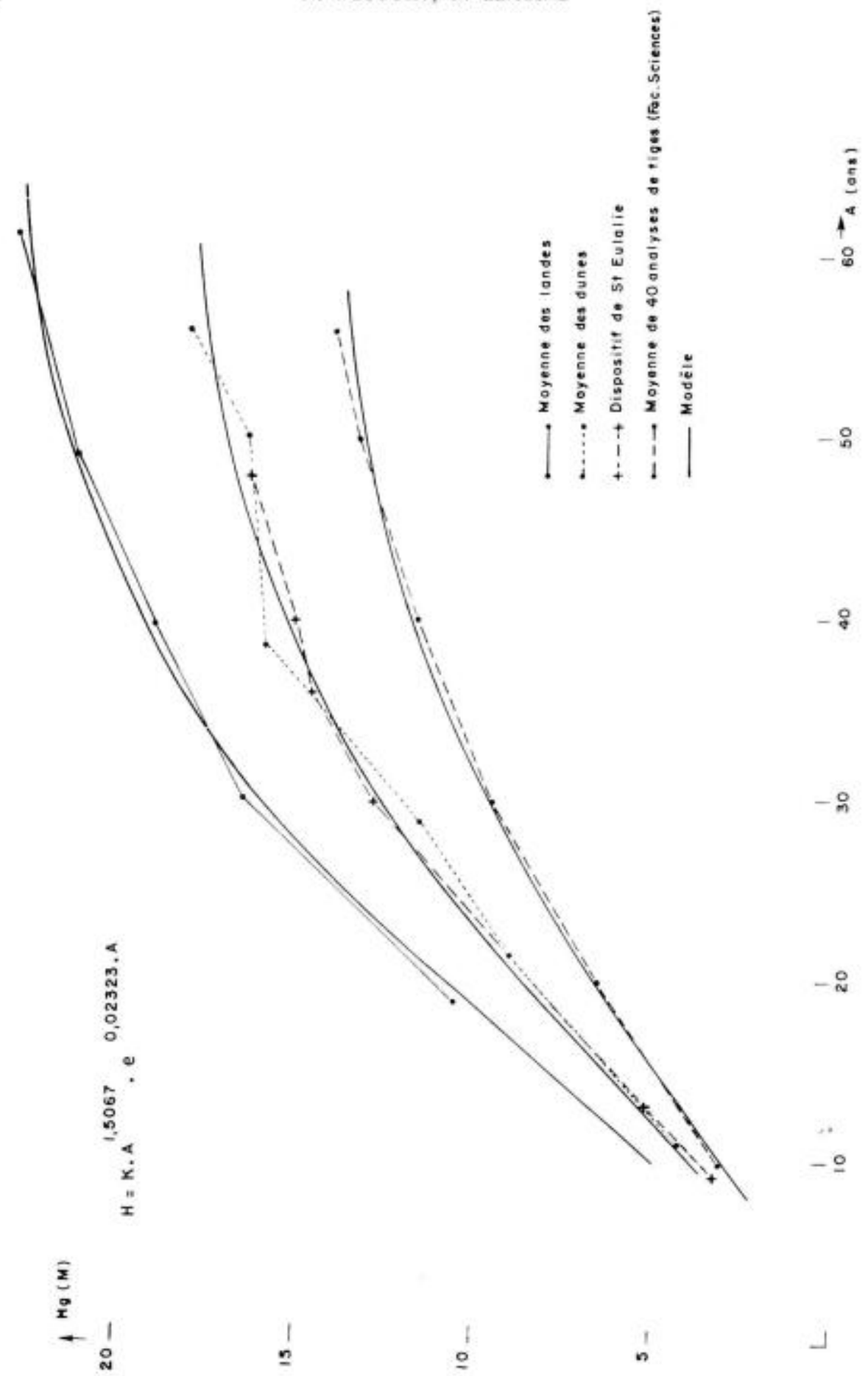

FIG. 4. - Vérification des courbes de croissance en hauteur 
Cela dit, quelle est la sylviculture la mieux adaptée au contexte économique actuel ? Nous ne pouvons répondre à cette question en toute rigueur. D'une part, nous anticiperions sur un des objets de nos recherches, et d'autre part, les expérimentations de Hourtin et de Ste-Eulalie furent installées dans une optique différente de celle que nous pouvons envisager aujourd'hui.

Ce qui était appelé " éclaircie faible $»$ il y a 20 ans ou 30 ans, nous paraît mériter l'appellation " éclaircie forte ", ce qui était appelé " éclaircie forte ", l'appellation " éclaircie extrêmement forte... voire abusive ».

Nous ne savons donc pas, a priori, quelle sera l'évolution des caractéristiques d'un peuplement sur lequel on aurait pratiqué une éclaircie plus faible que le niveau le plus faible pratiqué dans les placettes permanentes.

Rappelons rapidement les résultats donnés par ces dispositifs. La figure 2 et les tableaux 1 et 1 bis, nous permettent de faire les constatations suivantes :

- d'une part, il y a, entre les 2 types d'éclaircie, une différercz de production en volume bois tige très importante. Bien qu'il soit généralement admis pour bon nombre d'essences que la production totale varie peu dans un large éventail d'intensité d'éclaircie, pour le Pin maritime, il semble que les cclaircics couramment pratiquées se situent dans une zone oủ leur " intensité » agit nettement sur la production. Ces observations confirment pour l'essentiel les conclusions de l'étude de MAUge (1967) parue dans le bulletin de l'Afocel.

- d'autre part, le régime " d'éclaircic forte " occasionne un gain d'accroissement portant sur les circonférences moyennes et dominantes, ceci bien entendu par rapport au régime « éclaircie faible $»$ (voir tableaux 1 et 1 bis).

Dans l'expérimentation décrite par LEMOINE (1967), on trouve confirmation du premier point, à savoir que l'éclaircie forte occasionne une chute de production (dans ce cas de l'ordre de $15 \%$ ), mais non du second point, ce qui cst peut-être dû au fait que le couvert n'est pas encore refermé (les bandes sont encore bien apparentes).

A l'appui de toutes ces considérations et enseignements, cz qui va suivre va traiter du régime d'éclaircie que nous avons choisi. Nous allons en effet choisir pour notre table un régime d'éclaircie "raisonnable " ou "moyen " en nous appuyant sur les pratiques de sylviculteurs appliq̣uant dans la région une dectrine que nous savons suivie et réfléchie, et chez lesquels du reste nous disposons de placettes temporaires. Ces sylviculteurs sont : pour les dunes, l'Offic: nationa' dcs Forêts, pour les landes, les auteurs de " la Sylviculture moderne du Pin maritime dans les Landes de Gascogne n. (P. Lallemand, J.P. LeOnard, J. Martin, J.P. Mauge et R. Navarre, 1966).

\subsection{Intensité et nature des éclaircies}

Nous rappelons brièvement (DECOURT, 1965 et 1967) que pour nous :

- I'intensité de l'éclaircie est définie par le nombre de tiges laissées à l'hectare lorsque le peuplement atteint une hauteur donnée, 


\section{Tableau I}

Placettes permanentes de Hourtin

\begin{tabular}{|c|c|c|c|c|c|c|c|c|c|c|c|c|c|c|}
\hline \multicolumn{10}{|c|}{ Peuplement principal après éclaircie } & \multicolumn{3}{|c|}{ Eclaircies } & \multicolumn{2}{|c|}{$\begin{array}{l}\text { Production } \\
\text { totale }\end{array}$} \\
\hline $\begin{array}{l}\text { Placette et } \\
\text { traitement }\end{array}$ & $\begin{array}{l}\text { An- } \\
\text { nèe }\end{array}$ & $\begin{array}{c}\text { A } \\
\text { ans }\end{array}$ & $\mathrm{N}$ & $\underset{\left(\mathrm{m}^{2}\right)}{\mathbf{G}}$ & $\begin{array}{c}V \\
\left(m^{3}\right)\end{array}$ & $\begin{array}{l}\mathrm{CG} \\
(\mathrm{cm})\end{array}$ & $\begin{array}{l}\mathrm{HG} \\
(\mathrm{m})\end{array}$ & $\begin{array}{c}\mathrm{Co} \\
(\mathrm{cm})\end{array}$ & $\begin{array}{l}\text { Ho } \\
(\mathrm{m})\end{array}$ & $\underset{\left(m^{3}\right)}{\Sigma v}$ & $\frac{\frac{D v}{\Sigma i}}{(\%)}$ & $\begin{array}{l}\frac{\mathrm{vm}}{\mathrm{Vg}} \\
(\%)\end{array}$ & $\underset{\left(m^{3}\right)}{\text { II }}$ & $\begin{array}{l}\operatorname{Im} \\
\left(\mathrm{m}^{3}\right) \\
\text { an })\end{array}$ \\
\hline \multirow[t]{8}{*}{$\begin{array}{l}\mathrm{A}=\text { Eclaircie fai- } \\
\text { ble avec gemmage }\end{array}$} & 27 & 12 & 2404 & 6,52 & - & 18 & - & 32 & - & 4,3 & - & - & - & - \\
\hline & 31 & 16 & I 538 & 11,52 & - & 31 & - & 44 & - & 14,7 & - & - & - & \\
\hline & 36 & 21 & 988 & 14,82 & 63,6 & 43 & - & 57 & - & 36,3 & 36,3 & 68,7 & 999 & 4,8 \\
\hline & 47 & 32 & 552 & 18,06 & 106,9 & 64 & 12,0 & 77 & 12,8 & 94,2 & 46,8 & 80,2 & 201,1 & 6.3 \\
\hline & 53 & 38 & 452 & 19,51 & 142,1 & 74 & 13,5 & 87 & - & 113,7 & 44,4 & 66,6 & 255,8 & 6,7 \\
\hline & 57 & 42 & 356 & 18,13 & 131,3 & 80 & 14,1 & 92 & 15,6 & 137,3 & 51,1 & 71.7 & 268,6 & 6,4 \\
\hline & 61 & 46 & 320 & 19,39 & 141,5 & 87 & 14,5 & 99 & 15,6 & 147,5 & 51,0 & 63,3 & 289,0 & 6,3 \\
\hline & 65 & 50 & 320 & - & 149,6 & - & 15.1 & - & 16.2 & 147,5 & 49,6 & - & 297,1 & 5,9 \\
\hline \multirow{9}{*}{$\begin{array}{l}\mathbf{B}^{\prime}=\text { Eclaircie forte } \\
\text { avec gemmage }\end{array}$} & & & & & & & & & & & & & & \\
\hline & $\begin{array}{l}27 \\
31\end{array}$ & $\begin{array}{l}12 \\
16\end{array}$ & $\begin{array}{r}1.012 \\
668\end{array}$ & $\begin{array}{l}6,04 \\
9,08\end{array}$ & - & $\begin{array}{l}27 \\
41\end{array}$ & - & $\begin{array}{l}38 \\
51\end{array}$ & - & $\begin{array}{l}13,2 \\
25,5\end{array}$ & - & - & - & \\
\hline & 36 & 21 & 458 & 12,15 & 64,3 & 58 & - & 68 & - & 45,9 & 41,6 & 78,6 & 110,2 & 5,2 \\
\hline & 47 & 32 & 284 & 15,64 & 108,0 & ) 83 & 13,9 & 94 & 14,7 & 99,7 & 48,0 & 87.5 & 207.7 & 6.5 \\
\hline & 50 & 35 & 260 & 16,46 & 105,2 & 2. 89 & - & - & - & 107,9 & 50,6 & 85,5 & 213,1 & 6,1 \\
\hline & 53 & 38 & 190 & 14,58 & 101,4 & 98 & 15,3 & 105 & 16,6 & 136,9 & 57,4 & 82,6 & 238,3 & 6,3 \\
\hline & 57 & 42 & 188 & 16,32 & 120,0 & ) 104 & 16,1 & 111 & 17,1 & 137,9 & 53,4 & - & 257,9 & 6,1 \\
\hline & 61 & 46 & 188 & 18,65 & 148,7 & 112 & 17,1 & 124 & 18,4 & 137,9 & 58,2 & - & 236,6 & 6.2 \\
\hline & 65 & 50 & 188 & - & 169,7 & - & 17,5 & - & 18,0 & 137,9 & 44,8 & - & 307,6 & 6,2 \\
\hline \multirow{9}{*}{$\begin{array}{r}\mathrm{B}=\text { Eclaircie fai- } \\
\text { ble sans gemmage }\end{array}$} & & & & & & & & & & & & & & \\
\hline & 27 & 12 & 2480 & $\begin{array}{l}5,74 \\
9.26\end{array}$ & - & 17 & - & $\begin{array}{l}31 \\
42\end{array}$ & - & $\begin{array}{l}1,8 \\
9,4\end{array}$ & E & - & - & - \\
\hline & $\begin{array}{l}31 \\
36\end{array}$ & 21 & $\begin{array}{r}1514 \\
954\end{array}$ & $\begin{array}{r}9.20 \\
+13.52\end{array}$ & 58,8 & 342 & - & 56 & - & 27,3 & 31,7 & 62,3 & 86,1 & 4.1 \\
\hline & 47 & 32 & 566 & 19,12 & 115,8 & 65 & 11,8 & 81 & 12,9 & 73,3 & 38.7 & 69,9 & 189,1 & 5,9 \\
\hline & 50 & 35 & 550 & 21,30 & 130,4 & 470 & - & - & - & 76,7 & 37,0 & - & 207,1 & 5,9 \\
\hline & 53 & 38 & 430 & 14,86 & 132,4 & $\begin{array}{l}46 \\
+76\end{array}$ & 13,6 & 91 & 14,7 & 100,4 & 43,1 & 69,6 & 232,8 & 6,1 \\
\hline & 57 & 42 & 356 & 19.38 & 133,7 & 783 & 13,9 & 97 & 15,8 & 119,1 & 47.1 & 71,3 & 252,8 & 6.0 \\
\hline & 61 & 46 & 322 & 20,50 & 146,0 & ) 90 & 14,5 & 104 & 15,6 & 131,0 & 47,3 & 78,9 & 277,0 & 6,0 \\
\hline & 65 & 50 & 322 & 23,57 & 162,4 & 496 & 15,3 & 111 & 16,4 & 131,0 & 44,6 & $1-$ & 293,4 & 5.9 \\
\hline \multirow{9}{*}{$\begin{array}{l}A^{\prime}=\text { Eclaircie for- } \\
\text { te sans gemmage }\end{array}$} & & & & & & & & & & & & & & \\
\hline & $\begin{array}{l}27 \\
31\end{array}$ & $\begin{array}{l}12 \\
16\end{array}$ & $\begin{array}{r}1016 \\
664\end{array}$ & $\begin{array}{l}4,42 \\
7,66\end{array}$ & - & $\begin{array}{l}23 \\
38\end{array}$ & $E$ & $\begin{array}{l}35 \\
50\end{array}$ & $E$ & 18.2 & - & - & - & - \\
\hline & 36 & 21 & 460 & 9,60 & 42,8 & 351 & . & 62 & - & 31,7 & 42,5 & 78,0 & 74,5 & 3,5 \\
\hline & 47 & 32 & 282 & 14,04 & 94,8 & $\begin{array}{l}89 \\
\end{array}$ & 12,8 & 89 & 13,5 & 77,2 & 44,9 & 86,2 & 172,0 & 5,4 \\
\hline & 50 & 35 & 248 & 14,66 & 93.9 & $\begin{array}{l}96 \\
\end{array}$ & & 95 & - & 87,7 & 48.2 & 83,4 & 181,6 & 5,2 \\
\hline & 53 & 38 & 182 & 13,02 & 90,5 & $5 \quad 95$ & 14,8 & 101 & 15,3 & 114,2 & 56,3 & 85,1 & 202,7 & 5,4 \\
\hline & 57 & 42 & 178 & 15,02 & 108,0 & 103 & 15,6 & 110 & 16,2 & 115.8 & 51,7 & - & 223,8 & 5,3 \\
\hline & 61 & 46 & 178 & 17,77 & 134,0 & 112 & 16,1 & 119 & 16,9 & 115,8 & 46,3 & - & 249.8 & 5,4 \\
\hline & 65 & 50 & 173 & 19,80 & 154,5 & 5118 & 17,0 & 126 & 17,1 & 115,8 & 42,8 & - & 270,3 & 5,4 \\
\hline
\end{tabular}

- à intensité égale, la nature de l'éclaircie est précisée par le rapport $v_{m} / V_{g}$ où $v_{m}$ est le volume moyen de l'arbre enlevé en éclaircie et $V_{g}$ est le volume de l'arbre moyen du peuplement avant éclaircie.

La figure 5 donne l'évolution du nombre de tiges en fonction de la hauteur moyenne dans trois cas :

- dans les placettes permanentes de dunes, 
Tableau I bis

Placettes permanentes de Sainte-Eulalie

\begin{tabular}{|c|c|c|c|c|c|c|c|c|c|c|c|c|c|c|}
\hline \multicolumn{10}{|c|}{ Peuplement principal après éclaircie } & \multicolumn{3}{|c|}{ Eclaircies } & \multicolumn{2}{|c|}{$\begin{array}{l}\text { Production } \\
\text { totale }\end{array}$} \\
\hline $\begin{array}{l}\text { Placette et } \\
\text { traitement }\end{array}$ & $\begin{array}{l}\text { An- } \\
\text { née }\end{array}$ & $\begin{array}{c}\text { A } \\
\text { ans }\end{array}$ & $\mathrm{N}$ & $\begin{array}{l}\mathrm{G} \\
\left(\mathrm{m}^{2}\right)\end{array}$ & $\underset{\left(m^{3}\right)}{V}$ & $\begin{array}{l}\mathrm{CG} \\
(\mathrm{cm})\end{array}$ & $\begin{array}{l}\mathrm{HG} \\
(\mathrm{m})\end{array}$ & $\begin{array}{l}\mathrm{Co} \\
(\mathrm{cm})\end{array}$ & $\begin{array}{l}\text { Ho } \\
(\mathrm{m})\end{array}$ & $\sum_{\left(m^{3}\right)}^{\sum v}$ & $\left|\begin{array}{c}\Sigma v \\
\sum 1 \\
(0)\end{array}\right|$ & $\frac{\mathrm{vm}}{\overline{V g}}$ & $\underset{\left(m^{3}\right)}{\Sigma 1}$ & $\begin{array}{l}\operatorname{Im} \\
\left(\mathrm{m}^{3} /\right. \\
\text { an) }\end{array}$ \\
\hline $\begin{array}{l}\text { II A- Eclaircie for- } \\
\text { te avec gemmage }\end{array}$ & $\begin{array}{l}50 \\
53 \\
57 \\
61 \\
65\end{array}$ & $\begin{array}{l}33 \\
36 \\
40 \\
44 \\
48\end{array}$ & $\begin{array}{l}252 \\
180 \\
180 \\
178 \\
180\end{array}$ & $\begin{array}{c}15,09 \\
13,11 \\
15,53 \\
- \\
-\end{array}$ & $\begin{array}{r}98,5 \\
98,6 \\
117,9 \\
132,4 \\
148,7\end{array}$ & $\begin{array}{r}87 \\
96 \\
104 \\
- \\
\end{array}$ & $\begin{array}{l}\overline{14,4} \\
15,1 \\
15,9 \\
16,5\end{array}$ & $\begin{array}{l}- \\
- \\
-\end{array}$ & $\begin{array}{l}- \\
- \\
\overline{16,4} \\
16,9\end{array}$ & $\begin{array}{r}80,8 \\
105,5 \\
105,5 \\
105,5 \\
105,5\end{array}$ & $\begin{array}{l}45,1 \\
51,7 \\
47,2 \\
44,3 \\
41,5\end{array}$ & $\begin{array}{l}75,7 \\
70,1 \\
- \\
- \\
-\end{array}$ & $\begin{array}{l}179,3 \\
204,1 \\
223,4 \\
237,9 \\
254,2\end{array}$ & $\begin{array}{l}5,4 \\
5,7 \\
5,6 \\
5,4 \\
5,3\end{array}$ \\
\hline $\begin{array}{l}\text { III A- Eclaircie } \\
\text { Pallu avec gem- } \\
\text { mage }\end{array}$ & $\begin{array}{l}50 \\
53 \\
57 \\
61 \\
65\end{array}$ & $\begin{array}{l}33 \\
36 \\
40 \\
44 \\
48\end{array}$ & $\begin{array}{l}398 \\
300 \\
222 \\
222 \\
222\end{array}$ & $\begin{array}{c}19,64 \\
17,87 \\
16,15 \\
- \\
-\end{array}$ & $\begin{array}{l}124,6 \\
125,4 \\
119,3 \\
136,4 \\
144,1\end{array}$ & $\begin{array}{l}79 \\
87 \\
96 \\
- \\
-\end{array}$ & $\begin{array}{l}\overline{14,4} \\
14,8 \\
15,2 \\
15,6\end{array}$ & $\begin{array}{l}- \\
- \\
-\end{array}$ & $\begin{array}{l}- \\
- \\
\overline{15,9} \\
16,1\end{array}$ & $\begin{array}{r}63,4 \\
84,4 \\
111,1 \\
111,1 \\
111,1\end{array}$ & $\begin{array}{l}33,7 \\
40,2 \\
48,2 \\
44,8 \\
43,5\end{array}$ & $\begin{array}{l}69,3 \\
60,0 \\
70,3 \\
- \\
-\end{array}$ & $\begin{array}{l}188,0 \\
209,8 \\
230,4 \\
247,5 \\
255,2\end{array}$ & $\begin{array}{l}5,7 \\
5,8 \\
5,8 \\
5,6 \\
5,3\end{array}$ \\
\hline $\begin{array}{l}\text { I A- Eclaircie fai- } \\
\text { ble avec gemmage }\end{array}$ & $\begin{array}{l}50 \\
53 \\
57 \\
61 \\
65\end{array}$ & $\begin{array}{l}33 \\
36 \\
40 \\
44 \\
48\end{array}$ & $\begin{array}{l}600 \\
470 \\
356 \\
314 \\
312\end{array}$ & $\begin{array}{c}22,93 \\
22,18 \\
20,84 \\
21,52 \\
-\end{array}$ & $\begin{array}{l}146,1 \\
159,5 \\
149,6 \\
165,2 \\
175,5\end{array}$ & $\begin{array}{l}\overline{77} \\
86 \\
93 \\
-\end{array}$ & $\begin{array}{l}\overline{14,6} \\
15,1 \\
16,1 \\
16,8\end{array}$ & $\begin{array}{l}- \\
\overline{-} \\
104\end{array}$ & $\begin{array}{l}- \\
\bar{z} \\
17,0 \\
17,6\end{array}$ & $\begin{array}{l}102,2 \\
121,0 \\
152,0 \\
165,3 \\
165,3\end{array}$ & $\begin{array}{l}41,1 \\
43,1 \\
50,3 \\
50,0 \\
48,5\end{array}$ & $\begin{array}{l}83,9 \\
48,7 \\
70,8 \\
64,5 \\
\end{array}$ & $\begin{array}{l}248,3 \\
280,5 \\
301,6 \\
330,5 \\
340,8\end{array}$ & $\begin{array}{l}7,5 \\
7,8 \\
7,5 \\
7,5 \\
7,1\end{array}$ \\
\hline $\begin{array}{l}\text { II B- Eclaircie for- } \\
\text { te sans gemmage }\end{array}$ & $\begin{array}{l}50 \\
53 \\
57 \\
61 \\
65\end{array}$ & $\begin{array}{l}33 \\
36 \\
40 \\
44 \\
48\end{array}$ & $\begin{array}{l}270 \\
182 \\
182 \\
182 \\
182\end{array}$ & $\begin{array}{l}15,21 \\
12,44 \\
15,18 \\
17,12 \\
19,14\end{array}$ & $\begin{array}{r}118,1 \\
93,2 \\
115,1 \\
125,1 \\
138,2\end{array}$ & $\begin{array}{c}84 \\
93 \\
102 \\
109 \\
115\end{array}$ & $\begin{array}{l}\overline{14,2} \\
15,0 \\
16,0 \\
16,7\end{array}$ & $\begin{array}{l}- \\
- \\
118 \\
125\end{array}$ & $\begin{array}{l}- \\
- \\
\overline{16,9} \\
16,9\end{array}$ & $\begin{array}{r}92,3 \\
123,2 \\
123,2 \\
123,2 \\
123,2\end{array}$ & $\begin{array}{l}43,8 \\
56,9 \\
51,6 \\
49,6 \\
47,1\end{array}$ & $\begin{array}{c}76,1 \\
77,6 \\
- \\
- \\
-\end{array}$ & $\begin{array}{l}240,4 \\
216,4 \\
238,3 \\
248,3 \\
261,4\end{array}$ & $\begin{array}{l}6,4 \\
6,0 \\
5,9 \\
5,6 \\
5,4\end{array}$ \\
\hline $\begin{array}{l}\text { III B- Eclaircie } \\
\text { Pallu sans gem- } \\
\text { mage }\end{array}$ & $\begin{array}{l}50 \\
53 \\
57 \\
61 \\
65\end{array}$ & $\begin{array}{l}33 \\
36 \\
40 \\
44 \\
48\end{array}$ & $\begin{array}{l}402 \\
298 \\
224 \\
224 \\
224\end{array}$ & $\begin{array}{l}19,46 \\
16,79 \\
16,07 \\
18,43 \\
20,67\end{array}$ & $\begin{array}{l}122,8 \\
115,8 \\
118,3 \\
135,6 \\
155,2\end{array}$ & $\begin{array}{c}78 \\
84 \\
95 \\
102 \\
108\end{array}$ & $\begin{array}{l}\overline{14,2} \\
14,7 \\
15,8 \\
15,9\end{array}$ & $\begin{array}{l}- \\
\overline{-} \\
110 \\
117\end{array}$ & $\begin{array}{l}- \\
- \\
\overline{16,6} \\
16,8\end{array}$ & $\begin{array}{r}72,5 \\
102,6 \\
129,3 \\
129,3 \\
129,3\end{array}$ & $\begin{array}{l}37,1 \\
46,9 \\
52,2 \\
48,8 \\
45,4\end{array}$ & $\begin{array}{l}82,4 \\
83,3 \\
74,2 \\
- \\
-\end{array}$ & $\begin{array}{l}195,3 \\
218,4 \\
247,6 \\
264,9 \\
284,5\end{array}$ & $\begin{array}{l}5,9 \\
6,1 \\
6,2 \\
6,0 \\
5,9\end{array}$ \\
\hline $\begin{array}{l}\text { 1 B- Eclaircie fai- } \\
\text { ble sans gemmage }\end{array}$ & $\begin{array}{l}50 \\
53 \\
57 \\
61 \\
65\end{array}$ & $\begin{array}{l}33 \\
36 \\
40 \\
44 \\
48\end{array}$ & $\begin{array}{l}552 \\
468 \\
362 \\
320 \\
320\end{array}$ & $\begin{array}{l}22,75 \\
22,84 \\
22,20 \\
22,57 \\
26,10\end{array}$ & $\begin{array}{l}146,2 \\
164,2 \\
159,4 \\
169,0 \\
195,4\end{array}$ & $\begin{array}{c}\overline{78} \\
88 \\
94 \\
101\end{array}$ & $\begin{array}{l}\overline{14,7} \\
15,2 \\
16,5 \\
16,9\end{array}$ & $\begin{array}{l}- \\
- \\
\overline{107} \\
115\end{array}$ & $\begin{array}{l}- \\
\bar{z} \\
\overline{17,1} \\
18,1\end{array}$ & $\begin{array}{l}101,0 \\
117,8 \\
151,1 \\
169,6 \\
169,6\end{array}$ & $\begin{array}{l}40,8 \\
41,8 \\
48,6 \\
50,0 \\
46,5\end{array}$ & $\begin{array}{l}79,2 \\
61,0 \\
76,3 \\
85,0 \\
-\end{array}$ & $\begin{array}{l}247,2 \\
282,0 \\
310,5 \\
338,6 \\
365,0\end{array}$ & $\begin{array}{l}7,5 \\
7,8 \\
7,8 \\
7,7 \\
7,6\end{array}$ \\
\hline
\end{tabular}

- telle qu'elle est conseillée par P. Lallemand, J.P. Leonard, J. Martin, J.P. Mauge et R. Navarre (1966),

- telle qu'elle a été retenue pour nos tables.

Les états de densité relevés dans nos placettes temporaires situées en forêt de dunes se répartissent de part et d'autre de la " courbe éclaircie faible $»$ des placettes permanentes (fig. 5).

Les états de densité relevés dans nos placettes temporaires de Landes se répartissent de part et d'autre de la courbe d'évolution conseillée par les sylviculteurs landais susvisés, courbe qui parait donc représenter une sylviculture moyenne (fig. 5). 


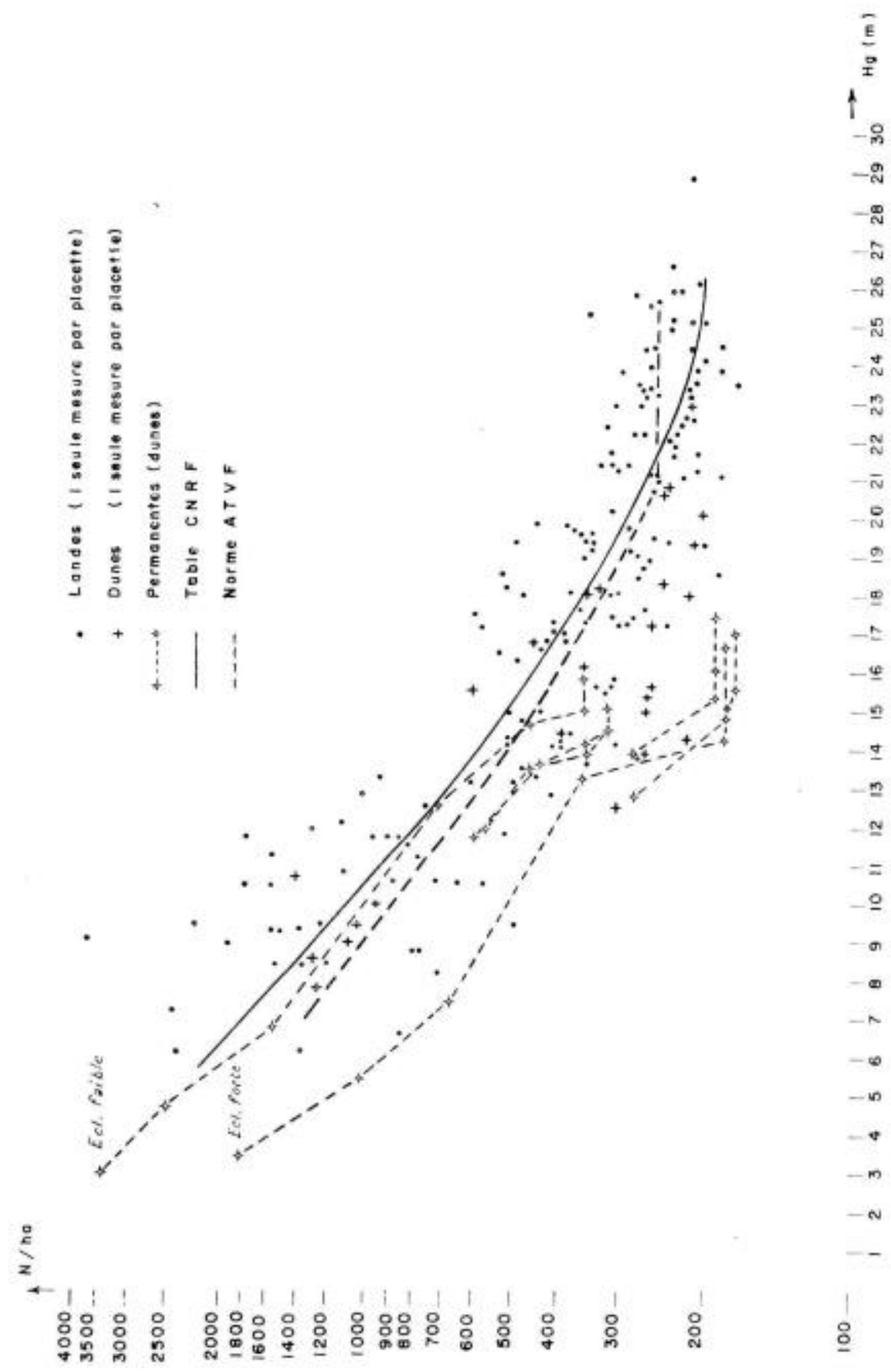

FIG. 5. - Nombre de tiges en fonction de la hauteur moyenne - Intensité de léclaircie 
Avant de décrire le régime d'éclaircie pour lequel nous avons opté, il nous parait utile de reproduire le texte suivant extrait du chapitre traitant de " la conduite des peuplements " (P. Lallemand, J.P. Leonard, J. Martin, J.P. Mauge et R. NAVARre, 1966).

"Cette essence ne permet donc pas une densité aussi importante que le Pin sylvestre ou les Pins Laricio et les éclaircies seront avant tout des éclaircies par le bas. Il faudra cependant travailler aussi l'étage dominant car le Pin maritime est un arbre à croissance rapide et il faut ménager à sa cime un espace vital suffisant pour lui permettre de s'étoffer tout en restant équilibré.

L'influence des éclaircies sur le Pin maritime est particulièrement sensible dans ses 25 ou 30 premières années.

Il sera nécessaire d'intervenir plus fréquemment et aussi plus énergiquement au début de cette période $n$.

Nous pensons en fait que cette observation sur le comportement en peuplement du Pin maritime est juste. La première conséquence de ceci est que l'on ne peut " travailler $n$ à coefficient d'espacement constant.

A ce titre, le régime (dit faible) d'éclaircie suivi dans la placette permanente de Ste-Eulalie jusqu'à une hauteur moyenne de $12,5 \mathrm{~m}$ semble convenir (ce régime est décrit dans la figure 5). Ensuite, le régime suivi nous paraît revêtir une particularité peu satisfaisante : on éclaircit brutalement deux fois et on n'intervient plus à partir d'une hauteur moyenne de $16 \mathrm{~m}$. Or, la plupart des peuplements (ceux des Landes en particulier) atteignent à 60 ans, voire à 50 et même 45 , des hauteurs bien supérieures à $16 \mathrm{~m}$. Quant aux auteurs cités ci-dessus, ils n'interviennent plus à partir d'une hauteur moyenne de $21 \mathrm{~m}$ (fig. 5). La classe 1 de Fertilité de notre table dépasse, au même âge, très nettement cette hauteur. Ne plus intervenir à partir de $21 \mathrm{~m}$ conduirait à ne plus passer en éclaircie pendant presque la moitié de la vie du peuplement.

C'est en fonction de toutes ces considérations et remarques que nous avons défini le régime d'éclaircie que nous croyons bon d'adonter. Il est décrit dans la figure 5 (voir également en annexe les tables de production).

La nature des éclaircies est fixé par le rapport $v_{m} / V_{y}$. L'étude de ce rapport dans un certain nombre de placettes temporaires (soit en forêt domaniale, soit en forêt privée) a permis de le relier de façon satisfaisante au nombre de tiges $N$, c'està-dire, implicitement à l'intensité de l'éclaircie.

On s'est aperçu de plus que les données relatives à la table espagnole (ECHEVERRIA et DE PEDRo, 1968) recoupaient et prolongeaient vers les fortes densités des données de nos placettes. Cette relation entre le type d'éclaircie et son intensité semble donc être une caractéristique de la sylviculture habituelle du Pin maritime.

\subsection{Rotations}

J. Guinaudeau et J. PARde (1963) adoptent une rotation quasiment uniforme de 4 ans pendant toute la vie du peuplement telle qu'elle est pratiquée dans les placettes permanentes. P. Lal.lemand, J.P. Leonard, J.P. Mauge et R. Navarre (1966) font varier la rotation qui est de 5 ans jusqu'à 30 ans, puis de 7 ans à partir de cet âge. 
Nous pensons qu'il est biologiquement justifié d'adopter une rotation variable avec l'âge. En effet, au début de sa vie, un peuplement croît plus vite qu'à la fin, il est donc logique d'espacer les interventions lorsque l'âge augmente.

Nous avons donc opté pour une rotation de 4 ans au début de la vie du peuplement, de 6 ans ensuite, de 8 ans à la fin (voir les tables). Nous terminerons sur ce point en soulignant que notre table traite uniquement de la Production en bois et non d'une Production mixte " bois-résine ». Si notre préoccupation avait été de rendre compte d'un type de Production avec récolte intensive de gemme, il est probable que nous eussions adopté une rotation uniforme de 4 ans.

Au demeurant, chacun pourra modifier les rotations en fonction des conditions locales... sans perdre de vue toutefois qu'il vaut mieux pour le bien devenir du peuplement principal pratiquer des éclaircies à des intervalles tels que l'on ne soit pas amené à intervenir par gros à-coups.

L'idéal serait évidemment d'espacer les interventions de telle sorte que la concurrence vitale n'apporte jamais aux arbres une gêne supérieure à une limite donnée à l'avance, de sorte que la croissance en diamètre soit constamment contrôlée par le sylviculteur. Des études plus précises sont nécessaires pour permettre un tel traitement et rien ne dit par ailleurs qu’il serait économiquement pratiquable.

\section{2. - Calcul de la table}

\subsection{Programme}

Toutes les données de base étant formalisées mathématiquement, un programme de calcul a été mis au point à la Station de Sylviculture et de Production pour permettre le calcul automatique sur ordinateur des tables de production. Il s'agit d'un programme écrit en ForTRAN IV (valable sur IBM 1130). On fournit à l'ordinateur toutes les relations fondamentales sous forme de "Subroutine $)$ ou de " fonction $)$. Les données se résument donc à celles qui sont nécessaires pour préciser le régime d'éclaircie désiré : nombre de tiges laissées sur pied pour une hauteur donnée du peuplement, succession des âges d'intervention.

L'ordinateur imprime une " table de base » ( ${ }^{1}$ ) correspondant au régime choisi ainsi que les tables de production habituelles réparties en classes de fertilité. On trouvera en annexe (cf. annexe 1), pour les Landes et pour les Dunes, les tables correspondant au traitement décrit ci-dessus (cf, 3.1.). Il va de soi, que nous pouvons à la demande et dans un délai très bref, fournir à l'utilisateur des tables construites sur le même modèle, mais correspondant à un autre traitement sylvicole.

\subsection{Limite de validité du modèle lors du choix d'un traitement sylvicole}

Rappelons que pour établir le modèle servant de base au calcul des tables, on s'appuie sur des relations fondamentales en principe indépendantes de la sylviculture pratiquée dans les placettes. Cela nous permet d'appliquer à ce modèle,

(1) Appelée * table intermédiaire * dans les publications précédentes (Decoukr 1965-1967). 
le traitement de notre choix, à condition que ce traitement ne diffère pas trop de ceux pratiqués effectivement dans l'ensemble des peuplements observés.

Ces limites sont décrites ci-dessous.

\section{- Intensité de l'éclaircie}

Le traitement peut laisser sur pied un nombre de tiges $N$ compris entre deux limites $N_{\text {inf }}$ et $N_{\text {sup }}$ variant avec la hauteur. On peut donner ces limites également en surface terrière, $G$ étant comprise entre $G_{\text {inf }}$ et $G_{\text {sup. }}$ tiges).

Le tableau ci-dessous donne un aperçu de ces limites (fig. 5, pour le nombre de

\begin{tabular}{|c|c|c|c|c|}
\hline $\begin{array}{c}H_{g} \\
(\mathrm{~m})\end{array}$ & $\begin{array}{l}N_{\text {inf }} \\
\text { (ha) }\end{array}$ & $\begin{array}{l}N_{s u p} \\
\text { (ha) }\end{array}$ & $\begin{array}{c}G_{i n f} \\
m^{2} / \text { ha) }\end{array}$ & $\begin{array}{c}G_{s u p} \\
\left(\mathrm{~m}^{2} / \mathrm{ha}\right.\end{array}$ \\
\hline 7,5 & 650 & 3500 & 7,00 & 19,0 \\
\hline 10,0 & 450 & 2000 & 9,50 & 29,0 \\
\hline 12,5 & 280 & 1300 & 12,00 & 31,5 \\
\hline 15,0 & 200 & 800 & 14,50 & 33,0 \\
\hline 17,5 & 190 & 600 & 17.50 & 35,0 \\
\hline 20,0 & 190 & 450 & 20,0 & 37,0 \\
\hline 22,5 & 180 & 350 & 23,0 & 38,0 \\
\hline 25,0 & 180 & 300 & 25,0 & 38,0 \\
\hline
\end{tabular}

\section{- Poids de chaque intervention}

Pour rester dans le cadre des observations faites, il faut également que le poids de chaque intervention ne dépasse pas une certaine limite. Autrement dit, il faut proscrire les interventions trop brutales. L'ensemble de nos placettes mesurées deux ou trois fois montre que le nombre de tiges enlevées lors d'une seule intervention se trouve assez généralement répartie entre 10 et $40 \%$ du nombre de tiges sur pied avant l'intervention. Cela, tant pour les dunes que pour les landes et quelle que soit la hauteur du peuplement (on peut même prendre zéro comme limite inférieure du fait de la disparition fréquente et souvent accidentelle d'un petit nombre d'arbres même dans les placettes non éclaircies). En volume, le poids d'une seule intervention se situe de la même façon entre 0 et $30 \%$ du matériel sur pied avant éclaircie.

\subsection{Précision des résultats}

Cette précision est difficile à estimer. Pour les peuplements sur pied, et à condition de se tenir dans les limites fixées ci-dessus, cette précision est assez satisfaisante. Elle est de l'ordre de celle permise par les équations fondamentales. 
Les productions et les accroissements, eux, sont liés au régime d'éclaircie suivi, la valeur des chiffres avancés dépend de celle du modèle. Il est pratiquement impossible de les tester autrement qu'en comparant les données des placettes permanentes avec les résultats théoriques, fournis par le modèle, pour des peuplements d'égale productivité et soumis à la même sylviculture. Pour les quelques placettes dont nous disposons, cette comparaison est satisfaisante. Les écarts constatés entre le modèle et la réalité ne dépassent pas $15 \%$ et sont souvent bien inférieurs pour la plupart des variables. Ces comparaisons sont cependant trop peu nombreuses pour qu'on puisse en tirer des résultats ayant une signification statistique. Disons seulement que les quelques vérifications faites sont satisfaisantes, car elles font ressortir une bonne concordancz entre ce qu'on observe sur le terrain et l'allure des phénomènes telle qu'elle est décrite par nos tables.

\section{IV. - TABLES ANNEXES}

Ces résultats annexes concernent :

- les découpes commerciales,

- le pourcentage d'écorce,

- le cubage rapide des peuplements sur pied,

- les correspondances entre la circonférence à $1,30 \mathrm{~m}$ et les circonférences à $3 \mathrm{~m}$ et $4 \mathrm{~m}$ de hauteur.

\section{1. - Découpes commerciales}

Le volume bois-fort tige (volume sur écorce à la découpe de $22 \mathrm{~cm}$ de circonférence au fin bout) est utilisé dans toutes les Stations de Recherche. Il est bien sûr nécessaire de mesurer une quantité bien définie, qui soit la même pour tous auin de rendre les résultats comparables. Ce volume ne correspond généralement pas aux découpes commerciales utilisées dans la pratique.

Nous avons done calculé les corrections nécessaires pour passer du volume bois-fort tige au volume sur écorce mais aux découpes $30 \mathrm{~cm}$ et $50 \mathrm{~cm}$ de circonférence.

On s'appuie sur les travaux de MAUGE (1961) qui a travaillé à partir de tiges mesurées soit dans les placettes permanentes de la Station de Recherches forestières de Bordeaux, soit dans certains peuplements du domaine de la Saussouze. II admet qu'une relation unique peut rendre compte de la forme moyenne du Pin maritime pour l'ensemble de la région.

Si $g$ est la surface de la section de la tige à la hauteur $h$, et si $g^{\prime}$ est la surface de la section à la mi-hauteur $H / 2$ de cette même tige, alors on aura :

$$
\frac{g}{g^{\prime}}=2-2 \frac{h}{H} .
$$


Cette relation est valable surtout dans la partie moyenne de la tige, et plus largement pour les $3 / 4$ supérieurs de celle-ci.

Les découpes que nous désirons étudier, se situant surtout dans cette zone, nous nous sommes appuyés sur cette équation (1). Pour chaque tige-échantillon de nos placettes, $H$ est connu ainsi que la section $g_{R F / 2}$ à la demi-hauteur bois-fort $\frac{H_{B F}}{2}$ on en tire facilement $g^{\prime}$ :

$$
g^{\prime}=\frac{1}{2} \frac{g H_{B r / 2} \times H}{H-\frac{H_{B F}}{2}} \quad \text { (voir équation 1) }
$$

Les volumes à la découpe donnée $g$, de hauteur $h$ seront cubés par la formule $V_{g}=h+g_{N / 2}$ où $g_{h / 2}$ est la section à la hauteur $h / 2$.

$$
\begin{aligned}
& \text { Or, on aura : } \quad g_{k_{j / 2}}=\frac{1}{2}\left(2 g^{\prime}+g\right) \\
& \text { et : } \quad h=\frac{2 g^{\prime}-g}{2 g^{\prime}} H \quad \text { (voir équation 1) } \\
& \text { Doou : } \quad V_{g}=\frac{1}{2} H \frac{\left(2 g^{\prime}-g\right)\left(2 g^{\prime}+g\right)}{2 g^{\prime}} \\
& V_{g}=\frac{1}{2} H \frac{4 g^{\prime 2}-g^{2}}{2 g^{\prime}} \text {. }
\end{aligned}
$$

Si $V_{T}$ est le volume total de la tige, cubé par la formule $V_{T}=H \times g^{\prime}$, on a alors :

$$
V_{v}=V_{T}-\frac{H}{4 g^{\prime}} g^{2} .
$$

Cela est vrai quelle que soit la découpe $g$, y compris si elle correspond à la découpe bois-fort $g_{B P}$. Cela entraîne avec le même système de notations :

$$
\begin{aligned}
& V_{S 0}=V_{B F}+\frac{i t}{4 g^{\prime}}\left(g_{B F}^{2}-g_{50}^{2}\right) \\
& V_{30}=V_{B F}+\frac{H}{4 g^{\prime}}\left(g_{B F}^{2}-g_{50}^{2}\right) .
\end{aligned}
$$

Les valeurs de $g^{\prime}, V_{50}, V_{30}$, ayant été calculées sur 84 tiges moyennes prélevées dans l'ensemble des placettes, on a pu ajuster les relations suivantes (le volume étant exprimé en $\mathrm{dm}^{3}$ ) :

$$
V_{50}=-88,915+1,056 V_{B F} ; R^{2}=0,998, \sigma=13,0
$$




$$
V_{30}=-11,211+1,011 V_{B F} ; R^{2}=0,999, \sigma=9,4
$$

Les coefficients de régression diffèrent légèrement mais significativement (au seuil $5 \%$ ) de 1 . Cela montre (cf. formules 3 et 4) que $H / g^{\prime}$ ne peut être considéré comme une constante. Effectivement, on constate que ce rapport croit légèrement avec la taille des arbres.

Ces relations sont cependant d'un emploi facile pour calculer les volumes aux découpes $50 \mathrm{~cm}$ et $30 \mathrm{~cm}\left(V_{50}\right.$ et $\left.V_{30}\right)$ d'un lot de $N$ tiges de volume bois-fort $V_{B F}$. On aura en effet (les volumes s'exprimant ici en $\mathrm{m}^{3}$ ) :

$$
\begin{aligned}
& V_{50}=-0,088915 \times N+1,056 V_{B F} \\
& V_{30}=-0,011211 \times N+1,011 V_{B F}
\end{aligned}
$$

Ces équations permettent de corriger les valeurs de la table, de façon approchée, tant pour les peuplements sur pied que pour les produits d'éclaircie.

\section{2. - Pourcentage d'écorce}

\subsection{Méthode d'évaluation et données recueillies}

Il convient d'associer tout particulièrement à cette étude, Monsieur l'Ingénieur du Génie rural des Eaux et des Forêts Dousset pour l'important travail qu'il a effectué sur le terrain et au bureau. Les mesures ont porté, par la méthode d'écorçage total, sur la tige entière ; 21 lots de pins abattus ont été mesurés de cette manière.

\subsection{Variations du pourcentage d'écorce}

Nous avons pu déceler deux causes de variations du pourcentage d'écorce :

a. sur une tige donnée : il varie suivant la position sur la tige, position que nous avons chiffrée en hauteur relative par rapport à la hauteur totale en partant du pied de l'arbre ;

b. d'une tige à l'autre ou d'un lot à l'autre : il varie suivant le volume $B F$ de la tige ou le volume $B F$ moyen du lot. La figure 6 rend compte des variations du pourcentage, de bois sans écorce par rapport au bois sur écorce.

1. le faisceau des courbes en pointillé fin représente la variation du pourcentage $b \%$, rapport des surfaces sous et sur écorce, d'une section en fonction de la hauteur relative où est située cette section et du volume de la tige.

2. la courbe en trait plein fort représente la variation du pourcentage $B \%$, rapport du volume de bois sous écorce au volume bois-fort sur écorce de l'arbre moyen d'un lot de pins. Si $Y$ est le volume de bois sous écorce correspondant au volume bois-fort $X$, on a pu calculer la relation donnant $Y$ en fonction de $X$ :

$$
Y_{\left(\mathrm{dm}^{3}\right)}=0,7501 X_{\left(\mathrm{dm}^{3}\right)}-11,2 .
$$




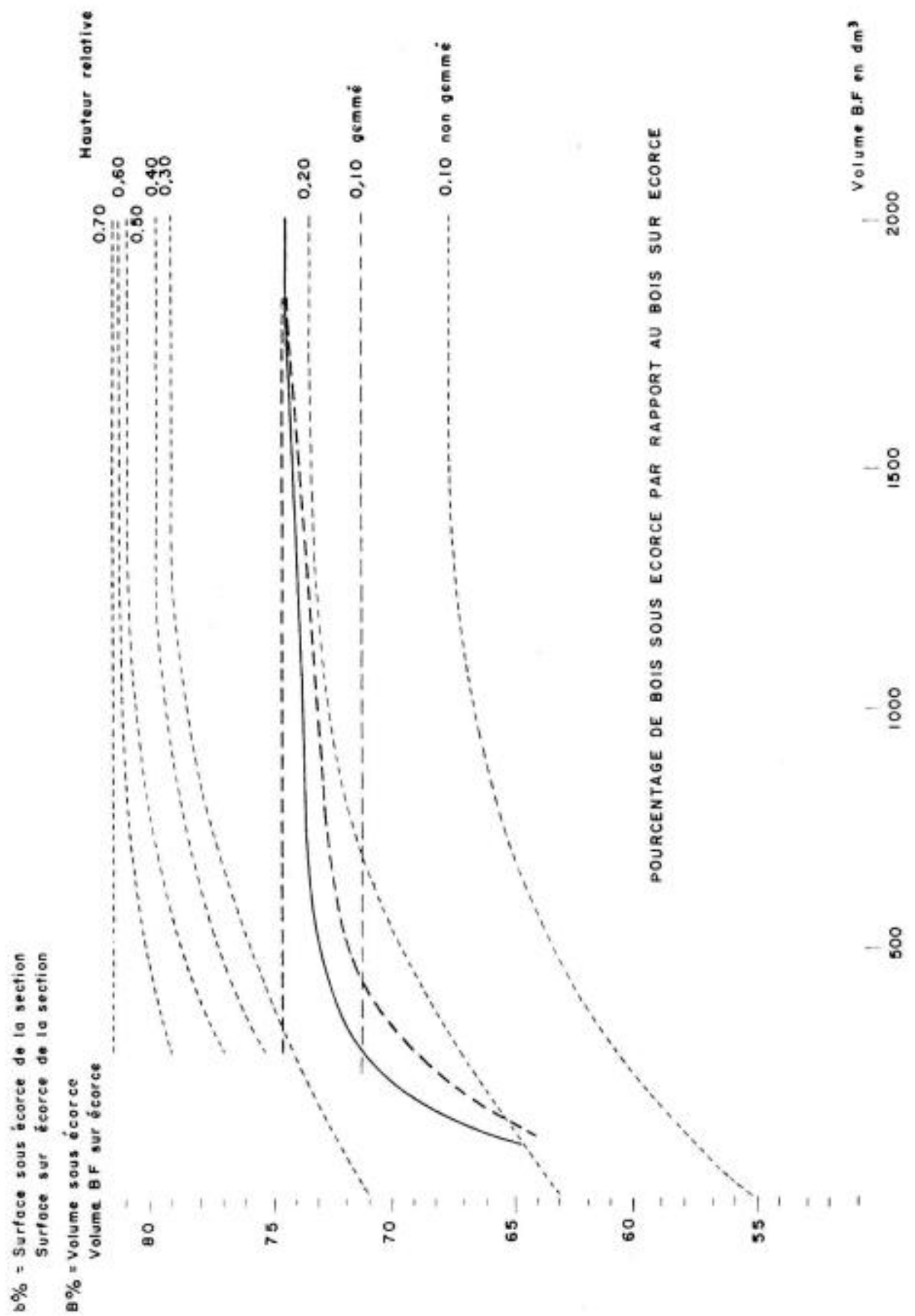

FiG. 6. - Pourcentage d'écorce moyen et à différents niveaux de hauteur 
La courbe de la figure 6 a été tracée point par point à partir de cette relation calculée à partir de lots de pins gemmés ou non gemmés. C'est cette courbe qu'il convient d'utiliser comme complément à la table de production et à la fonction de cubage traduite en abaque.

Pour un lot de $N$ tiges, de volume bois-fort sur écorce $V_{B F}$ et de volume sous écorce $V_{B F E}$, on aura (les volumes étant exprimés en $\mathrm{m}^{3}$ ) :

$$
V_{B F B}=0,7501 V_{B N}-11,2 \cdot 10^{-3} \times N
$$

Les courbes en tiretés forts sont destinées à montrer l'effet du gemmage sur le pourcentage d'écorce. Elles ont été construites à partir des courbes $b \%$ et de l'équation générale donnant la forme de la tige (MAugE, 1961).

La courbe supérieure (arbres gemmés) a pu pratiquement être assimilée à une droite : autrement dit, on ne relève pas, contrairement à ce qui se passe pour les arbres non gemmés (courbe inférieure), de variation du pourcentage $B \%$ en fonction des différentes valeurs du volume moyen de la tige.

Pour en revenir à l'étude sur les coefficients $b \%$, il est bien évident qu'elle constitue un complément à l'étude sur les découpes. Signalons cependant que le pourcentage d'écorce relatif à la découpe $50 \mathrm{~cm}$ de tour, n'est inférieur que de $1 \%$ à celui correspondant à la découpe bois-fort $(22 \mathrm{~cm})$. La correction est donc inutile aux découpes commerciales usuelles.

\section{3. - Cubage des peuplements}

La construction de la table de production utilise une relation de la forme :

$$
\log _{e} V_{\left(m^{3} / \mathrm{ba}\right)}=a+b \log _{e} G_{\left(m^{2} /(t a)\right.}+c \log _{e} H_{y(m)} .
$$

On peut mettre à profit l'existencz de cette relation pour effectuer un cubage rapide des peuplements sur pied.

Le volume calculé a partir de cette relation est connu avec une erreur type relative de l'ordre de $5 \%$.

A partir de ces équations peu maniables, nous avons construit 2 abaques (voir annexe 3) suivant le principe exposé par ARBONNIER (1958). Notons que les 3 axes sont orientés dans le même sens et qu'y sont reportées en longueur les valeurs successives de $(\log V-a)$, de $\left(b \log _{e} G\right)$ et de $\left(c \log _{e} H_{g}\right)$. La lecture se fait évidemment en $H, G$ et $V$ dont les valeurs sont graduées directement sur les 3 axes.

Nous rappelons pour l'utilisation de cet abaque, qu'il suffit de tracer la droite reliant les points situés sur les 2 axes extérieurs aux valeurs de $H$ et de $G$ mesurées sur le terrain : le point d'interscction de catte droite et de l'axe médian donne la valeur de $V$ cherchée.

En ce qui concerne les mesures à effectuer sur le terrain :

1. pour évaluer la surface terrière et la circonférence moyenne $C_{g}$, on effectuera bien entendu, un inventaire du peuplement en circonférence (ou en diamètre) ; 
2. la mesure des hauteurs totales de 5 à 6 arbres de circonférence moyenne permet d'évaluer la hauteur $H_{g}$ (par simple moyenne arithmétique de ces quelques mesures).

\section{4. - Cas des peuplements gemmés - Correspondance entre la circonférence à $1,30 \mathrm{~m}$ et les circonférences à $3 \mathrm{~m}$ et $4 \mathrm{~m}$}

Dans le cas des peuplements gemmés, la mesure des circonférences à $1,30 \mathrm{~m}$ est rendue imprécise par la présence des cares. Dans ce cas, il est possible d'estimer la grosseur des arbres en mesurant les circonférences à $3 \mathrm{~m}\left(C_{3,0}\right)$ ou à $4 \mathrm{~m}\left(C_{4,0}\right)$. Nous avons d'ailleurs pratiqué ainsi, dans nos placettes pour évaluer les volumes sur pied d'un certain nombre de peuplements. 48 mesures ont été faites à $3 \mathrm{~m}$ et 30 à $4 \mathrm{~m}$. La table de production ne donne que des circonférences à $1,30 \mathrm{~m}$. Pour le calcul de ces tables, les relations faisant intervenir la surface terrière ou la circonférence ont donc été établies sur des peuplements non gemmés, ou suffisamment peu gemmés pour que les mesures à $1,30 \mathrm{~m}$ restent valables. On a ensuite recherché une liaison entre $C_{1,30} ; C_{3,0}$ et $C_{4,0}$. L'équation tarif établie à partir de mesures à $1,30 V=f\left(G, H_{g}\right)$ a permis en effet, connaissant $V$ et $H_{g}$ de calculer la surface terrière théorique $G_{1,30}$ à $1,30 \mathrm{~m}$ dans les peuplements gemmés ; et donc, la circonférence moyenne théorique $C_{1,30}$ correspondant à la hauteur observée et au volume évalué à partir de mesures à $3,0 \mathrm{~m}$ ou à $4,0 \mathrm{~m}$ :

$$
\left.C_{1,30}=C_{3,0} \sqrt{\frac{G_{1,30}}{G_{3,0}}} \text { (de même à partir de } C_{4,0}\right) \text {. }
$$

D'excellentes relations linéaires relient, comme on s'y attendait, ces circonférences à $1,30 \mathrm{~m}$ reconstituées et les circonférences mesurées à $3,0 \mathrm{~m}$ ou à $4,0 \mathrm{~m}$ :

$$
\begin{aligned}
& C_{1,30}=9,0786+1,0786 C_{4,0} ; R^{2}=0,985, \sigma=1,66 \\
& C_{1,30}=0,0194+1,1129 C_{3,0} ; R^{2}=0,964, \sigma=2,67
\end{aligned}
$$

Connaissant $C_{4,0}$ ou $C_{3,0}$, il est donc facile d'évaluer très précisément $C_{1,30}$. Ces relations sont valables tant pour les peuplements de Dunes que pour ceux de Landes (figure de l'Annexe 4).

\section{V. - INTENSITÉ D'ÉCLAIRCIE ET PRODUCTION}

Le modèle adopté rend bien compte des remarques faites au $\$ 3.1$., remarques fondées sur les résultats observés dans divers dispositifs expérimentaux. Plus l'éclaircie est forte, plus la production totale est faible (fig. 2). 

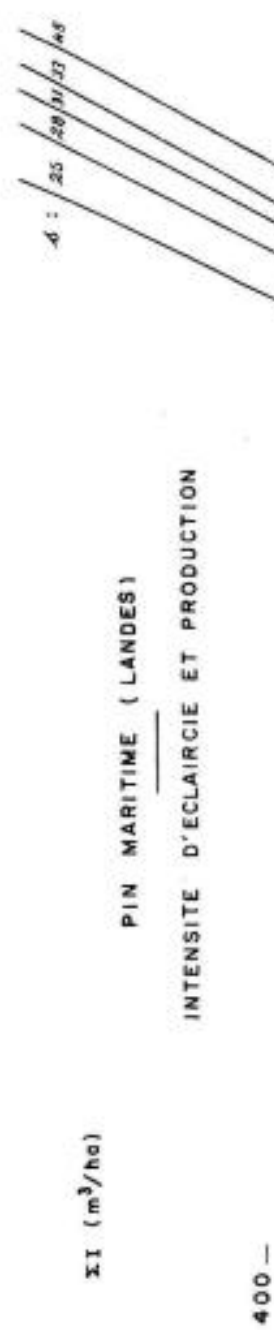

!

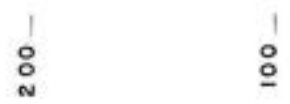

$\overline{0}$

Fig. 7 et 7 bis. - Production totale et intensité des éclaircies mesurées par le facteur de Hart-Becking $(s \%)$ 


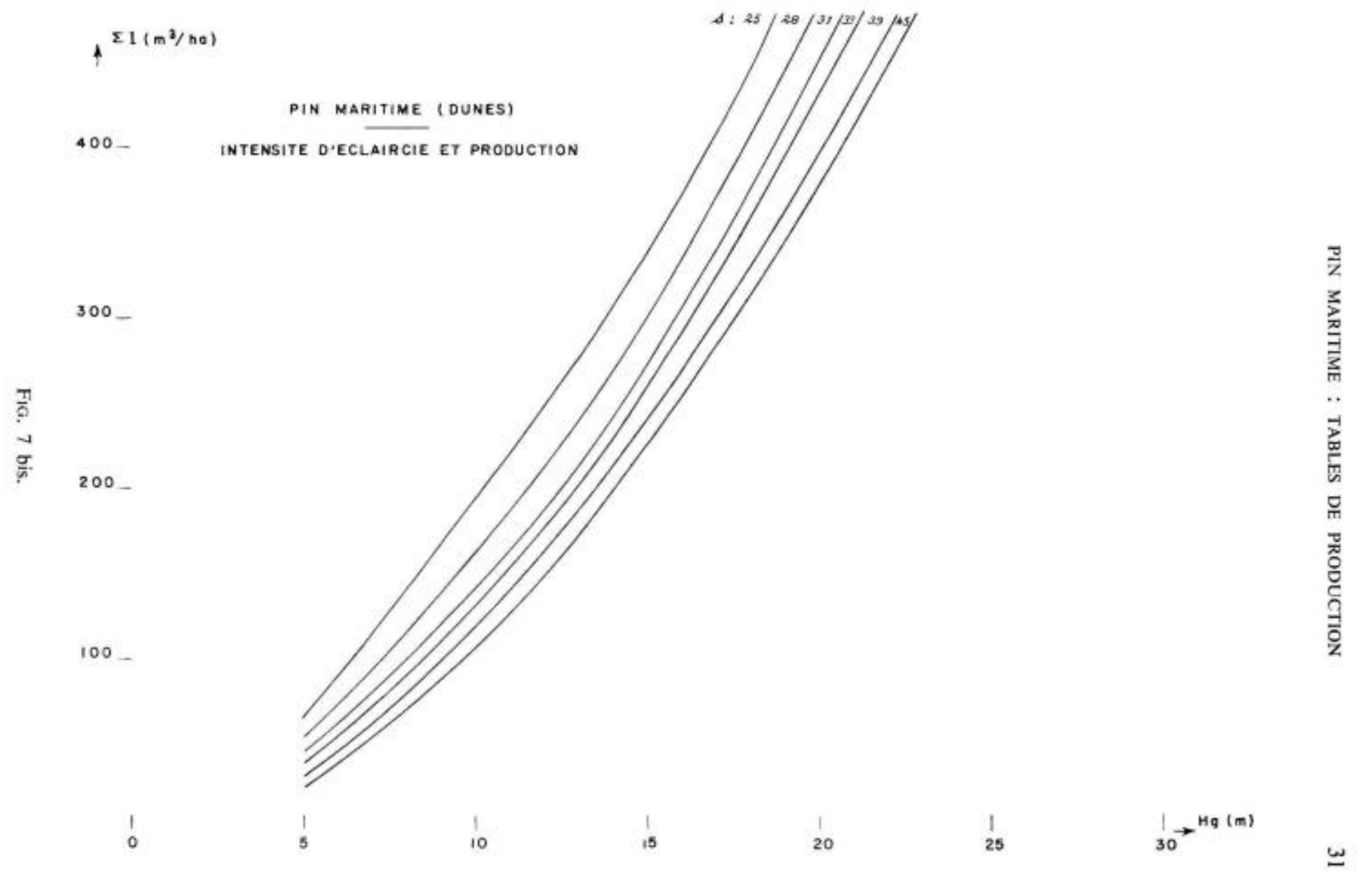


Les figures 7 et 7 bis, montrent comment, selon nos tables, la production totale varie avec l'intensité des éclaircies pratiquées ; les régimes théoriques comparés sont caractérisés par des facteurs d'espacements différents, maintenus constants pendant toute la vie du peuplement. Le facteur d'espacement en cause est ici celui de HART-BECKING qui exprime en pourcentage de la hauteur dominante, l'espacement moyen des tiges supposées régulièrement réparties aux nœuds d'un réseau fait de triangles équilatéraux (BECKING, 1953 ; PARDE, 1961). On constate un effet considérable de la densité du peuplement sur la production.

Le même effet, bien sûr, peut être observé sur l'accroissement courant annuel en volume $\left(I_{c}\right)$. MAUGE (1967) constate dans son dispositif clinal d'ANDERNos, une chute d'accroissement pratiquement linéaire lorsque la surface terrière laissée sur pied $(G)$ du peuplement diminue. Nous avons voulu vérifier que notre modèle de croissance rendait bien compte de cet effet.

Il suffit pour cela de partir de nos équations fondamentales (cf. tabl. 2) :

$$
\begin{aligned}
\log V & =a+b \log G+c \log H_{g} \\
C_{g} & =a^{\prime}+b^{\prime} H_{g}+c^{\prime} / N
\end{aligned}
$$

avec bien entendu :

$$
G=\frac{N C_{g}^{2}}{4 \pi} .
$$

On tire de ces équations :

et

$$
\begin{aligned}
& \frac{\mathrm{d} V}{\mathrm{~d} H_{g}}=b \frac{\mathrm{d} G}{\mathrm{~d} H_{g}} \frac{V}{G}+c \frac{V}{H_{g}} \\
& \frac{\mathrm{d} V}{\mathrm{~d} H_{g}}=V\left(\frac{2 b b^{\prime}}{C_{g}}+\frac{c}{H_{g}}\right) .
\end{aligned}
$$

Connaissant par ailleurs $(\$ 2.24$. $)$ :

$$
\frac{\mathrm{d} H_{y}}{\mathrm{~d} A}=a^{\prime \prime}+\frac{b^{\prime}}{A}
$$

on aura facilement :

$$
I_{c}=\frac{\mathrm{d} V}{\mathrm{~d} A}=\frac{\mathrm{d} V}{\mathrm{~d} H_{g}} \frac{\mathrm{d} H_{g}}{\mathrm{~d} A} .
$$

Le calcul montre effectivement que dans les limites de validité de nos équations les variations de $I_{c}$ en fonction de $G$ sont représentées par des courbes très tendues, à concavité tournée vers le bas, mais très voisines de droites. 


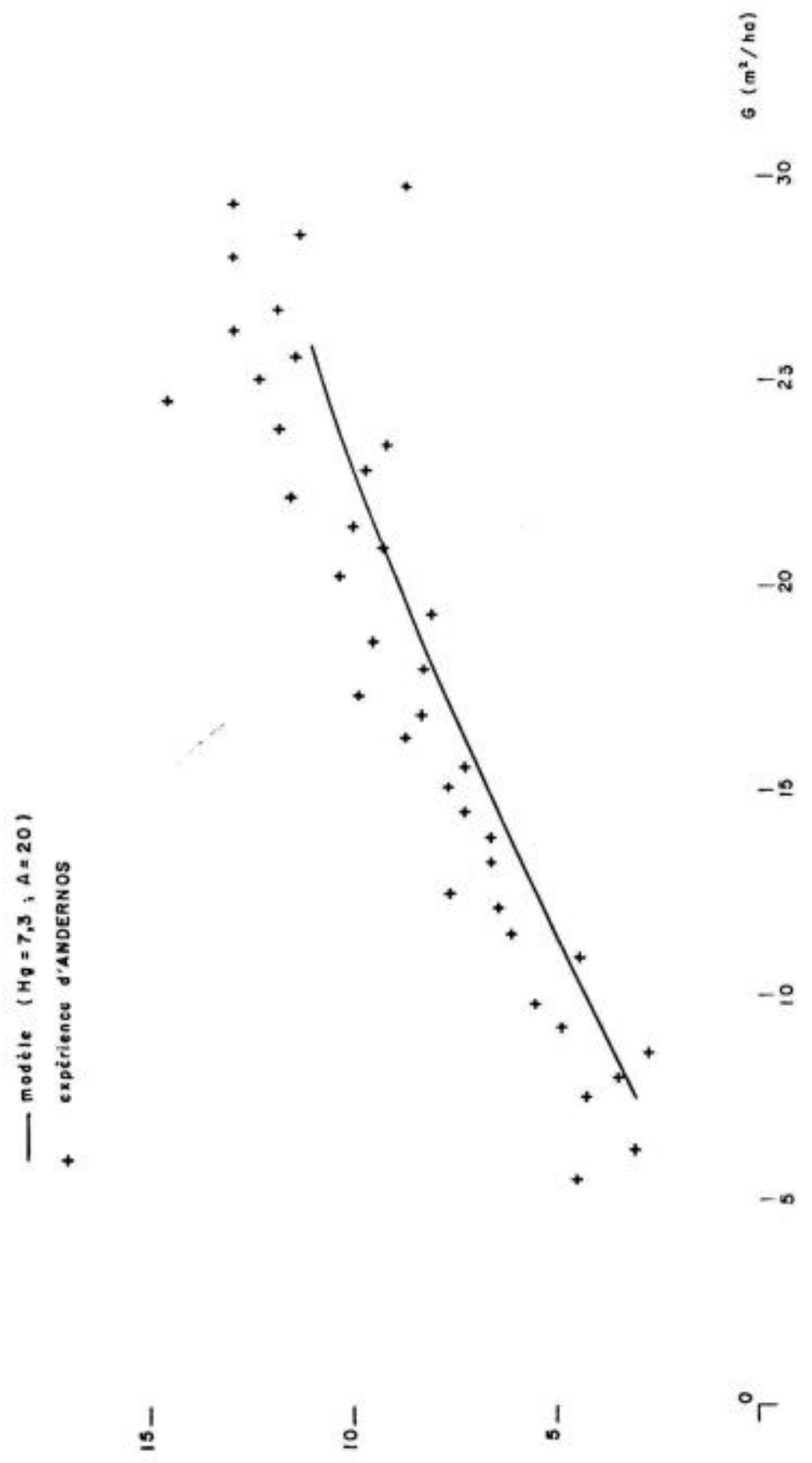

FIG. 8. - Variation de l'accroissement courant en volume en fonction de la surface terrière - Comparaison du modẻle et des résultats obtenus à Andernos 
Si on applique ces équations au peuplement d'ANDERNOS pour lequel $H_{g} \simeq 7,3 \mathrm{~m}$ et $A \simeq 20$ ans, la courbe obtenue est assimilable à une droite traversant le nuage de points donné par MAUGE de façon très satisfaisante (fig. 8).

Les chiffres donnés par nos tables ne correspondent donc qu’à la sylviculture qui s'y trouve décrite. Les éclaircies plus fortes notamment, entrainent une diminution de production notable, dont notre modèle rend compte, et qui se trouve confirmée par l'observation.

\section{VI. - CONCLUSION}

Ces tables, pour des raisons évidentes, ne sont pas définitives. Elles constituent une première approche objective du problème.

Nous pensons en effet que :

1. il nous faudra encore beaucoup de mesures répétées dans nos placettes pour construire de véritables fonctions de croissance valables dans des conditions écologiques données et variées ;

2. le choix d'un régime d'éclaircie convenable devra s'appuyer sur des résultats expérimentaux plus nombreux. Ceci est reporté à une échéance plus lointaine. Un certain nombre d'expérimentations destinées à répondre à ces questions sont en cours.

Le régime d'éclaircie proposé correspond à une sylviculture réellement pratiquée dans bon nombre de nos placettes. II correspond à des éclaircies sensiblement moins fortes que celles traditionnellement en usage dans la région. Cela conduit, en fonction de considérations économiques, à substituer à une sylviculture mixte " bois-gemme $)$ une sylviculture plus nettement orientée vers la production de bois.

Reçu pour publication en novembre 1968

\section{REMERCIEMENTS}

Nous tenons à remercier :

- les propriétaires particuliers qui nous ont autorisés à installer chez eux nos placettes et sans lesquels le présent travail n'aurait pu voir le jour, car la forêt landaise est constituée comme on le sait pour plus des $7 / 10$ de propriétés privées.

- l'Office National des Forêts pour l'aide que nous ont apportée ses agents.

- MM. Parde et Guinaudeau, respectivement directeurs de la Station de Sylviculture et de Production et de la Station de Recherches forestieres de Bordeaux, pour leurs nombreux et excellents conseils.

- le département de Biométrie qui a pris en charge les calculs de régressions progressives et qui nous a facilité l'accession aux moyens de calcul et la mise au point de nos programmes, ainsi que M. AYraL, calculateur à la Station de Sylviculture et de Production qui a effectué une part importante des calculs.

— Enfin, et tout particulièrement Monsieur I'Ingénieur du G.R.E.F. Dousser, qui a participé activement à l'installation de la majorité des placettes temporaires utilisées avec une compétence et une connaissance du terrain extrếmement précieuse. 


\section{SUMMARY \\ Maritime Pine (Pinus pinaster Art.) In the SOUth West of France. TEMPORARY YIELD TABLES}

The yield tables are concerned with a very important species in the Southwest of France where it covers about 1000000 ha. They were calculated almost completely with data obtained from temporary plots which were mesured one, sometimes twice.

The method used basically a certain number of fundamental equations which describe the state and the growth of the stand. These equations were obtained by multiple regressions. From these equations, and after having chosen a treatment (intensity, nature and period of the thinnings) we could built a model simulating the evolution of stands which have been treated in that way. The tables we are writing here are thus for variable thinnings regime.

First of all these tables indicate that overthinnings decrease obviously the yield of the Pine stands. In this study we only considered the production of wood, and not that of resin. This is the reason why the thinnings are less heavy than those usually practiced in that area.

Besides, the environment has a certain influence upon the growth of Pine. We had to distinguish two kinds of stands and to build two sets of tables : one for the stands growing on the heath and one for the stands growing on the dunes. Studies in progress will give more details about these differences and will permit to improve the tables.

\section{ZUSAMMENFASSUNG}

\section{Die Sefstrandkiffer (Pinus pinaster Ait.) im SüdWesten Frankri.ichs VORLÄUFIGE ERTHAGSTAFELN}

Es wurden Ertragstafeln für die Seestrandkiefer (Pinus pinaster ArT.) erstellt, welehe in Södwestfrankreich einen bedeutenden Platz einnimmt und einen einheitlichen Waldkomplex von ungefähr 1 Million Hektar bildet.

Die Tafeln wurden nahezu ausschliesslich mit ein oder zweimal aufgenommenen «temporären " Probeflächen berechnet. Die Methode beruht auf der Berechnung von mehreren Grundgleichungen (Mehrfachregressionsanalyse) welche den Zustand und das Wachstum der Bestände beschreiben. Mit Hilfe dieser Gleichungen und nach Wahl einer bestimmten waldbaulichen Behandlung (Durchforstungsart - Stärke und - Turnus), kann ein Modell erstellt werden, das die entsprechende Bestandesentwicklung wiedergibt. Es handelt sich daher um Ertragstafeln mit variablen Durchforstungsregime.

Als ein wesentliches Ergebnis konnte gezeigt werden, dass bei zu starker Durchforstung die Gesamtmassenleistung der Seestrandkiefer wesentlich abnimmt. Der Ertragstafel wurde als Produktionsziel die Holzerzeugung und nicht die Harznutzung zu Grunde gelegt, sodass die gewăhlte Durchforstungsstārke geringer ist, als es bisher in dieser Region allgemein üblich war.

Desweiteren zeigte sich, dass der Standort einen nicht zu vernachlässigenden Einfluss auf den Wachstumsgang der Seestrandkiefer ausübt. Es war daher notwendig zwei Ertragstafeln zu erstellen, welche als eine erste vorläufige Standortklassifizierung den Unterschieden zwischen Beständen der "Landes " und den Dünenbeständen Rechnung tragen. Bereits laufende Untersuchungen zur genaueren Unterscheidung der Standortseinheiten werden eine spätere Verbesserung der Tafeln ermöglichen.

\section{RÉFÉRENCES BIBLIOGRAPHIQUES}

ArbonNIER P., 1958. - Une méthode graphique pour la détermination rapide du volume approché d'un peuplement. Rev. forest. fr., déc. 796-806.

Beckıng J.M., 1953. - Einige Gesichtspunkte für die Durchfürung von vergleichenden Durchforstungsversuchen in gleichältrigen Beständen. Rapport du $11^{\circ}$ Congrès de II.U.F.R.O. Rome 580-582.

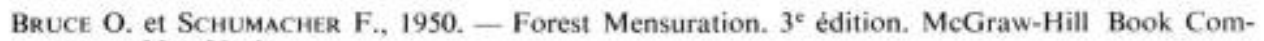
pany, New York. 
Claverie P.A.. 1958. - «Le Pin des Landes ou Pin maritime au cours de l'histoire ». Bulletin société Borda, Dax, $1^{\text {er }}$ trimestre, 75-91.

Curtis R.O., 1964. - A Stem-Analysis Approach to Site-Index Curves. Forest Scĩ., 10 (2), 241-256.

DAvid R., 1961. - « La croissance du Pin maritime dans diverses localités du Massif forestier Landais ", Rec, Trav, Lab. Biol, vég. Fac. Sci. Bordeaux, 2-49,

DAvid R., 1967. - «Influence du gemmage à l'acide sulfurique sur l'activité histogène du Cambium de la tige principale du Pin maritime : comparaison avec le gemmage au Hapchott 3 . Rec. Trav, Lab. Biol, vég. Fac. Sci. Bordeaux 6. C. R. Acad. Sci. Paris, 265, 1606-1611.

Dfcourt N., 1965. - Le Pin sylvestre et le Pin Laricio de Corse en Sologne, Tables de production provisoires et méthodes utilisées pour les construire. Amm. Sci. forest., 22 (2), 359-318.

Decourt N., 1967. - Le Douglas dans le Nord-Est du Massif Central. Tables de Production provisoires, Amm. Sci, forest., 24 (1), $45-84$.

Echeverria I. et De Pedro S. 1948, - El Pinus Pinaster en Pontevedra. Instinus Forestal de Investigaciones y experiencias. Madrid, 1948.

Enjalbert H., 1950. - « Observations morphologiques sur les Landes de Gascogne », Rev, géogr. Pyrénées Sud-Ouest, 21 (1) p. 42.

Guinaudeau J., 1964. - "La forêt landaise ". I.N.R.A. Stn Rech. forest. Bordeaux. Ronéo,

Guinaudeau J.. et Parde J., 1963, - " Contribution à létude de la sylviculture et de la production du Pin maritime (Pinus pinaster Sol,) dans les Landes, Notes techn. forest. 17. Sin. Rech. Exper. forest. 14, rue Girardet 54-Nancy.

Guinaudeau J., Illy G., Mauge J.P. et Dumas F., 1963. - Essai de Fertilisation Minérale sur Pin maritime à Mimizan (Landes). Amm. Ec, nation, Eaux Foréts, 22 (1) 1-71.

ILLY G., 1966, - « Recherches sur l'amélioration génétique du pin maritime n. Am. Sci. forest., 23 (4) $769-944$.

Inventaire Forestier Namoval. - Départements des Landes, de la Gironde et du Lot-et-Garonne Minist. Agric, Direcrion des Foréts et de TEspace Rural.

Lallemand P., Leonard J.P. et Martin J., 1960. - La Sylviculture moderne du Pin maritime dans les Landes de Gascogne. Bull techn. Inform. Ingrs. Serv, agric., 150, 16 p.

Lallemand P., Leonard J.P. Martin J., Maugie. J.P. et Navarre R., 1966. - La Sylviculture moderne du Pin maritime dans les Landes de Gascogne. Bull, techn. Inform. Ingrs. Serv. agric., 208 et $210,30 p$.

LANDES dE Gascogne, 1948. - "Compte rendu du sommaire des études et travaux effectués en application de l'ordonnance du 28 avril 1945. Les Frères Douladonne, Toulouse, 29 cartes.

L.fMOINE B., 1967. - Les dispositifs modernes de sylviculture du Pin maritime dans les Landes de Gascogne, Premiers résultats d'un dispositif factoriel. Rapp, $14^{\circ}$ Congr I.U.F.R.O. (Munich) 4, 353-370,

Mauge J.P., 1961. - Une nouvelle méthode de cubage du Pin maritime pour la région landaise. Ann. Ec, Eaux Forêts. Nancy, 18, 71-95.

Mauge J.P., 1967. - Expérience d'Eclaircie dans un peuplement de Pin maritime de 20 ans à Andernos (Gironde). C.R. activité 1967. AFOCEL, 102-120.

MARTin G., 1965, - Analyse de régresssions multiples. Thèse for. Nancy.

Oudin A., 1938, - Etudes sur le gemmage des Pins en France», Amn. Sin Rech. Exper. forest. Nancy, 7, 167-291.

PARDE J., 1961. - Comment préciser l'intensité d'une éclaircie, Rev, forest. fr. Août-sept. 551-5 57.

SArgos R., 1949. - «Contribution à I'histoire du boisement des Landes de Gascogne ". Editions Delmas, Bordeaux, $836 \mathrm{p}$.

Sargos R., 1954. - « Les Landes de Gascogne et la culture du Pin maritime », 305 p. Imp. J. Lacoste Mont-de-Marsan (Landes).

Scotr C.W., 1962. - « A Summary on information on Pinus Pinaster ». Forestry abstr., 23 (1 et 2). \$ 1-8 et $9-17$.

TOMASsONE R., 1967, - Régression progressive multiple. Programme FORTRAN 67 009. Sin Biométrie, INRA-Nancy.

Tomassone R., et Miluer E., 1968, - Eléments d'Analyse Statistique à plusieurs variabl es. CH 2. p. 55. Séminaire de Biométrie de IINRA. Mars 1968. 
Table de production pour le Pin maritime da sud-ouest de la France (Landes)

\begin{tabular}{|c|c|c|c|c|c|c|c|c|c|c|c|c|c|c|c|c|c|}
\hline \multicolumn{9}{|c|}{ PEUPLEMENT PRINCIPAL. } & \multicolumn{9}{|c|}{ ECLAIRCIES } \\
\hline $\begin{array}{l}\text { Age } \\
\text { Ans }\end{array}$ & $\begin{array}{c}\text { Hdom } \\
\mathrm{M}\end{array}$ & $\underset{\mathrm{M}}{\mathrm{Hmoy}}$ & Tiges & $\underset{\mathrm{CM}}{\mathrm{Cm}}$ & $\begin{array}{c}\text { Cdom } \\
\text { CM }\end{array}$ & $\underset{\mathrm{M} 3}{\mathrm{Vmoy}}$ & $\begin{array}{l}\text { St } \\
\text { M2 }\end{array}$ & $\begin{array}{l}\text { Vol } \\
\text { M3 }\end{array}$ & Tiges & $\begin{array}{c}\text { Cmoy } \\
\mathrm{CM}\end{array}$ & $\begin{array}{c}\text { Vmoy } \\
\text { M3 }\end{array}$ & $\begin{array}{l}\text { Vol } \\
\text { M3 }\end{array}$ & $\begin{array}{l}\text { Vtot } \\
\text { M3 }\end{array}$ & $\begin{array}{c}\text { Ecl } \\
100\end{array}$ & $\begin{array}{c}\text { Prtot } \\
\text { M3 }\end{array}$ & $\begin{array}{c}\text { Ace,c } \\
M 3 / A n\end{array}$ & $\begin{array}{l}\text { Acc.m } \\
\text { M3/An }\end{array}$ \\
\hline \multicolumn{18}{|c|}{ Classe 1} \\
\hline 12 & 9,3 & 7,4 & 1658 & 31,7 & 50,5 & 0,027 & 13,3 & 46.2 & 0 & 0.0 & 0,000 & 0,0 & 0,0 & 0,0 & 46.2 & - & 3,8 \\
\hline 16 & 12,2 & 10,5 & 1021 & 46,9 & 66,0 & 0,080 & 17,9 & 82,4 & 637 & 33,4 & 0,039 & 25,0 & 25,0 & 23,3 & 107,5 & 16.2 & 6,7 \\
\hline 20 & 14,9 & 13,4 & 642 & 63,2 & 82,2 & 0,182 & 20,4 & 117,5 & 379 & 48,5 & 0,088 & 33,7 & 58,8 & 33,3 & 176,3 & 18,5 & 8,8 \\
\hline 24 & 17,5 & 16,1 & 441 & 79,8 & 98,4 & 0,349 & 22,3 & 154,2 & 201 & 64,1 & 0.213 & 42,9 & 101,7 & 39,7 & 255,9 & 19,8 & 10,6 \\
\hline 28 & 19,8 & 18,5 & 341 & 94,9 & 113,1 & 0,563 & 24,4 & 192,2 & 100 & 77,8 & 0.414 & 41,4 & 143,2 & 42,6 & 335,4 & 19.1 & 11,9 \\
\hline 34 & 22,7 & 21,5 & 255 & 115,3 & 132,8 & 0,958 & 26,9 & 244,5 & 86 & 94,9 & 0,696 & 59,9 & 203,1 & 45,3 & 447,6 & 16,7 & 13,1 \\
\hline 40 & 25,0 & 23.9 & 223 & 129,5 & 146.7 & 1,341 & 29,7 & 299,4 & 32 & 107,6 & 1,068 & 34,1 & 237,3 & 44,2 & 536,7 & 12,9 & 13,4 \\
\hline 46 & 26,7 & 25,7 & 207 & 139,3 & 156,5 & 1,658 & 32.0 & 344,3 & 16 & 116,6 & 1.336 & 21,3 & 258,6 & 42,9 & 603,0 & 8,9 & 13,1 \\
\hline 54 & 28,1 & 27,2 & 198 & 147,1 & 164,4 & 1.944 & 34,1 & 385,2 & 9 & 123,8 & 1,941 & 17,4 & 275,1 & 41,7 & 661.4 & 5,1 & 12,2 \\
\hline 62 & 28,6 & 27,8 & 194 & 150,3 & 167.7 & 2,072 & 34,9 & 402,3 & 4 & 126,8 & 1.817 & 7,2 & 283,4 & 41,3 & 685,7 & - & 11,0 \\
\hline \multicolumn{18}{|c|}{ Classe 2 , } \\
\hline 12 & 8,4 & 6,5 & 1899 & 27,4 & 46,0 & 0,018 & 11,3 & 35,6 & 0 & 0,0 & 0,000 & 0,0 & 0,0 & 0.0 & 35,6 & - & 2,9 \\
\hline 16 & 10.9 & 9,2 & 1266 & 40,1 & 59,1 & 0,052 & 16,2 & 66,3 & 633 & 27,3 & 0,023 & 14,8 & 14,8 & 18.2 & 81,1 & 12,3 & 5,0 \\
\hline 20 & 13,3 & 11.7 & 828 & 53,7 & 72,8 & 0,117 & 19,0 & 97,2 & 438 & 39,5 & 0,050 & 22,1 & 37,0 & 27,5 & 134,2 & 14,0 & 6,7 \\
\hline 24 & 15,6 & 14,1 & 573 & 67,4 & 86,3 & 0,219 & 20,7 & 125.7 & 255 & 52,1 & 0,120 & 30.8 & 67.8 & 35,0 & 193,5 & 15,5 & 8,0 \\
\hline 28 & 17,6 & 16,2 & 435 & 80,4 & 99,0 & 0,357 & 22.4 & 155,5 & 138 & 64,7 & 0,257 & 35,4 & 103,3 & 39.9 & 258,8 & 15,4 & 9,2 \\
\hline 34 & 20,1 & 18.8 & 328 & 97,3 & 115,4 & 0,604 & 24,7 & 198,1 & 107 & 79.9 & 0.432 & 46,2 & 149,5 & 43,0 & 347.7 & 13,8 & 10,2 \\
\hline 40 & 22,1 & 20,9 & 270 & 111,1 & 128,8 & 0,866 & 26,5 & 234,1 & 58 & 91,6 & 0,713 & 41,3 & 190,9 & 44,9 & 425,0 & 11.3 & 10,6 \\
\hline 46 & 23,6 & 22,5 & 236 & 121,6 & 138,8 & 1,113 & 27,8 & 263,6 & 34 & 100,1 & 0,846 & 28,7 & 219.7 & 45.4 & 483,3 & 7,5 & 10.5 \\
\hline 54 & 24,8 & 23,8 & 224 & 128,6 & 145,9 & 1,315 & 29.5 & 295,5 & 12 & 106.8 & 1,307 & 15,6 & 235,3 & 44,3 & 530,9 & 4,2 & 9,0 \\
\hline 62 & 25,3 & 24,3 & 219 & 131.6 & 148,8 & 1,406 & 30,2 & 308,8 & 5 & 109,5 & 1,306 & 6,5 & 241,9 & 43,9 & 550,7 & - & 8.8 \\
\hline \multicolumn{18}{|c|}{ Classe 3} \\
\hline 12 & 7,5 & 5,6 & 2140 & 23,2 & 41,7 & 0,011 & 9,1 & 25,0 & 0 & 0,0 & 0,000 & 0,0 & 0,0 & 0,0 & 25,0 & - & 2,0 \\
\hline 16 & 9,7 & 7,9 & 1553 & 33,7 & 52,6 & 0,032 & 14,0 & 50,8 & 587 & 19,8 & 0,013 & 7,6 & 7,6 & 13,0 & 58,4 & 9,1 & 3,6 \\
\hline 20 & 11,7 & 10.0 & 1106 & 44,5 & 63,6 & 0,069 & 17,4 & 76.8 & 447 & 31,3 & 0,031 & 13,8 & 21,5 & 21,8 & 98,3 & 10,5 & 4,9 \\
\hline 24 & 13,7 & 12,0 & 792 & 55,4 & 74.5 & 0,127 & 19,4 & 101,2 & 314 & 41,2 & 0,062 & 19,7 & 41,2 & 28,9 & 142,5 & 11,1 & 5,9 \\
\hline 28 & 15,4 & 13,8 & 591 & 66,2 & 85,1 & 0,208 & 20.6 & 123,0 & 201 & 51.0 & 0,116 & 23,4 & 64.7 & 34,4 & 187,7 & 11.5 & 6,7 \\
\hline 34 & 17,6 & 16,1 & 436 & 80,3 & 98,9 & 0,355 & 22,4 & 155,3 & 155 & 64,6 & 0,247 & 38,3 & 103,0 & 39,8 & 258,3 & 10,8 & 7,5 \\
\hline 40 & 19,3 & 17,9 & 360 & 91,5 & 109,8 & 0.510 & 24,0 & 183,6 & 76 & 74,8 & 0,407 & 30,9 & 134,0 & 42,1 & 317,6 & 8,6 & 7.9 \\
\hline 46 & 20,5 & 19,3 & 312 & 100,3 & 118,3 & 0,656 & 25.0 & 205,1 & 48 & 82,3 & 0.478 & 22,9 & 157,0 & 43,3 & 362,1 & 6,1 & 7,8 \\
\hline 54 & 21,6 & 20.4 & 284 & 107,3 & 125,1 & 0,789 & 26,0 & 224.3 & 28 & 88,5 & 0,800 & 22.4 & 1794 & 44,4 & 403,7 & 3.6 & 7,4 \\
\hline 62 & 22,0 & 20,8 & 272 & 110.4 & 128,1 & 0.852 & 26,4 & 132,3 & 12 & 91,0 & 0,784 & 9,4 & 188,8 & 44,8 & 421,1 & - & 6.7 \\
\hline
\end{tabular}


Table de production pour le Pin maritime du sud-ouest de la France (Landes)

\begin{tabular}{|c|c|c|c|c|c|c|c|c|c|c|c|c|c|c|c|c|c|}
\hline \multicolumn{9}{|c|}{ PEUPLEMENT PRINCIPAL } & \multicolumn{9}{|c|}{ ECLAIRCIES } \\
\hline $\begin{array}{l}\text { Age } \\
\text { Ans }\end{array}$ & $\begin{array}{c}\text { Hdom } \\
\mathrm{M}\end{array}$ & $\underset{\mathrm{M}}{\mathrm{Hmoy}}$ & Tiges & $\underset{\mathrm{CM}}{\mathrm{Cmoy}}$ & $\begin{array}{c}\text { Cdom } \\
\mathrm{CM}\end{array}$ & $\begin{array}{c}\mathrm{Vmoy} \\
\mathrm{M} 3\end{array}$ & $\begin{array}{l}\mathrm{St} \\
\mathrm{M} 2\end{array}$ & $\begin{array}{l}\text { Vol } \\
\text { M3 }\end{array}$ & Tiges & $\begin{array}{c}\text { Cmoy } \\
\mathrm{CM}\end{array}$ & $\begin{array}{c}\text { Vmoy } \\
\text { M3 }\end{array}$ & $\begin{array}{l}\text { Vol } \\
\text { M3 }\end{array}$ & $\begin{array}{l}\text { Vtot } \\
\text { M3 }\end{array}$ & $\begin{array}{c}\text { Ecl } \\
/ 100\end{array}$ & $\begin{array}{c}\text { Prtot } \\
\text { M3 }\end{array}$ & $\begin{array}{c}\text { Acc.c } \\
\text { M3/An }\end{array}$ & $\begin{array}{l}\text { Acc.m } \\
\text { M3/An }\end{array}$ \\
\hline \multicolumn{18}{|c|}{ Classe 4} \\
\hline $\begin{array}{l}12 \\
16 \\
20 \\
24 \\
28 \\
34 \\
40 \\
46 \\
54 \\
62\end{array}$ & $\begin{array}{r}6,6 \\
8,4 \\
10,1 \\
11,7 \\
13,2 \\
15,0 \\
16,4 \\
17,5 \\
18,3 \\
18,7\end{array}$ & $\begin{array}{r}4,6 \\
6,5 \\
8,3 \\
10,0 \\
11,5 \\
13,4 \\
14,9 \\
16,1 \\
17,0 \\
17,4\end{array}$ & $\begin{array}{r}2300 \\
1892 \\
1425 \\
1107 \\
848 \\
635 \\
514 \\
442 \\
394 \\
380\end{array}$ & $\begin{array}{l}19,2 \\
27,5 \\
36,1 \\
44,5 \\
52,7 \\
63,6 \\
72,6 \\
79,7 \\
85,6 \\
87,9\end{array}$ & $\begin{array}{r}37,4 \\
46,2 \\
55,1 \\
63,5 \\
71,8 \\
82,6 \\
91,5 \\
98,3 \\
104,1 \\
106,3\end{array}$ & $\begin{array}{l}0,006 \\
0,019 \\
0,039 \\
0,069 \\
0,112 \\
0,185 \\
0,268 \\
0,348 \\
0,425 \\
0,457\end{array}$ & $\begin{array}{r}6,7 \\
11,4 \\
14,8 \\
17,4 \\
18,8 \\
20,5 \\
21,6 \\
22,3 \\
23,0 \\
23,4\end{array}$ & $\begin{array}{r}15,8 \\
35,9 \\
56,4 \\
76,7 \\
95,1 \\
118,2 \\
138,3 \\
154,0 \\
167,9 \\
174,1\end{array}$ & $\begin{array}{r}0 \\
408 \\
467 \\
318 \\
259 \\
213 \\
121 \\
72 \\
48 \\
14\end{array}$ & $\begin{array}{r}0,0 \\
10,8 \\
23,2 \\
31,3 \\
38,5 \\
48,8 \\
57,5 \\
64,0 \\
69,4 \\
71.6\end{array}$ & $\begin{array}{l}0,000 \\
0,010 \\
0,010 \\
0,039 \\
0,050 \\
0,116 \\
0,192 \\
0,258 \\
0,327 \\
0,475\end{array}$ & $\begin{array}{r}0,0 \\
4,1 \\
4,7 \\
12,5 \\
13,1 \\
24,8 \\
23,3 \\
18,6 \\
15,7 \\
6,6\end{array}$ & $\begin{array}{r}0,0 \\
4,1 \\
8,9 \\
21,4 \\
34,6 \\
59,5 \\
82,8 \\
101,5 \\
117,2 \\
123,8\end{array}$ & $\begin{array}{r}0,0 \\
10,3 \\
13,6 \\
21,8 \\
26,7 \\
33,4 \\
37,4 \\
39,7 \\
41,1 \\
41.5\end{array}$ & $\begin{array}{r}15,8 \\
40,1 \\
65,4 \\
98,2 \\
129,7 \\
177,7 \\
221,2 \\
255,5 \\
285,1 \\
298,0\end{array}$ & $\begin{array}{l}-6,1 \\
7,2 \\
8,0 \\
7,9 \\
7,6 \\
6,4 \\
4,5 \\
2,6\end{array}$ & $\begin{array}{l}1,3 \\
2,5 \\
3,2 \\
4,0 \\
4,6 \\
5,2 \\
5,5 \\
5,5 \\
5,2 \\
4,8\end{array}$ \\
\hline \multicolumn{18}{|c|}{ Classe 5} \\
\hline $\begin{array}{l}12 \\
16 \\
20 \\
24 \\
28 \\
34 \\
40 \\
46 \\
54 \\
62\end{array}$ & $\begin{array}{r}5,7 \\
7,2 \\
8,5 \\
9,8 \\
11,0 \\
12,4 \\
13,6 \\
14,4 \\
15,1 \\
15,4\end{array}$ & $\begin{array}{r}3,7 \\
5,2 \\
6,7 \\
8,0 \\
9,2 \\
10,7 \\
11,9 \\
12,8 \\
13,6 \\
13,9\end{array}$ & $\begin{array}{r}2300 \\
2230 \\
1857 \\
1513 \\
1258 \\
973 \\
802 \\
703 \\
621 \\
587\end{array}$ & $\begin{array}{l}15,5 \\
21,6 \\
28,1 \\
34,5 \\
40,3 \\
48,4 \\
55,0 \\
59,9 \\
64,4 \\
66,4\end{array}$ & $\begin{array}{l}33,4 \\
40,0 \\
46,8 \\
53,3 \\
59,4 \\
67,5 \\
74,0 \\
79,0 \\
83,4 \\
85,3\end{array}$ & $\begin{array}{l}0,004 \\
0,009 \\
0,020 \\
0,034 \\
0,053 \\
0,087 \\
0,124 \\
0,157 \\
0,192 \\
0,210\end{array}$ & $\begin{array}{r}4,4 \\
8,3 \\
11,7 \\
14,3 \\
16,3 \\
18,1 \\
19,3 \\
20,1 \\
20,5 \\
20,6\end{array}$ & $\begin{array}{r}9,4 \\
21,0 \\
37,4 \\
52,6 \\
66,9 \\
85,6 \\
100,1 \\
110,8 \\
119,7 \\
123,4\end{array}$ & $\begin{array}{r}0 \\
70 \\
373 \\
344 \\
255 \\
285 \\
171 \\
99 \\
82 \\
34\end{array}$ & $\begin{array}{r}0,0 \\
1,8 \\
11,7 \\
20,9 \\
27,5 \\
34,6 \\
40,8 \\
45,5 \\
49,5 \\
51,1\end{array}$ & $\begin{array}{l}0,000 \\
0,010 \\
0,010 \\
0,010 \\
0,027 \\
0,041 \\
0,075 \\
0,116 \\
0,116 \\
0,117\end{array}$ & $\begin{array}{r}0,0 \\
0,7 \\
3,8 \\
3,5 \\
7,1 \\
11,9 \\
12,9 \\
11,4 \\
9,5 \\
3,9\end{array}$ & $\begin{array}{r}0,0 \\
0,7 \\
4,5 \\
8,0 \\
15,1 \\
27,1 \\
40,1 \\
51,5 \\
61,1 \\
65,1\end{array}$ & $\begin{array}{r}0,0 \\
3,2 \\
10,7 \\
13,2 \\
18,4 \\
24,0 \\
28,5 \\
31,7 \\
33,8 \\
34,5\end{array}$ & $\begin{array}{r}9,4 \\
21,7 \\
41,9 \\
60,6 \\
82,0 \\
112,7 \\
140,2 \\
162,4 \\
180,9 \\
188,6\end{array}$ & $\begin{array}{l}\overline{4,0} \\
4,8 \\
5,0 \\
5,2 \\
4,8 \\
4,1 \\
2,9 \\
1,6 \\
-\end{array}$ & $\begin{array}{l}0,7 \\
1,3 \\
2,0 \\
2,5 \\
2,9 \\
3,3 \\
3,5 \\
3,5 \\
3,3 \\
3,0\end{array}$ \\
\hline
\end{tabular}




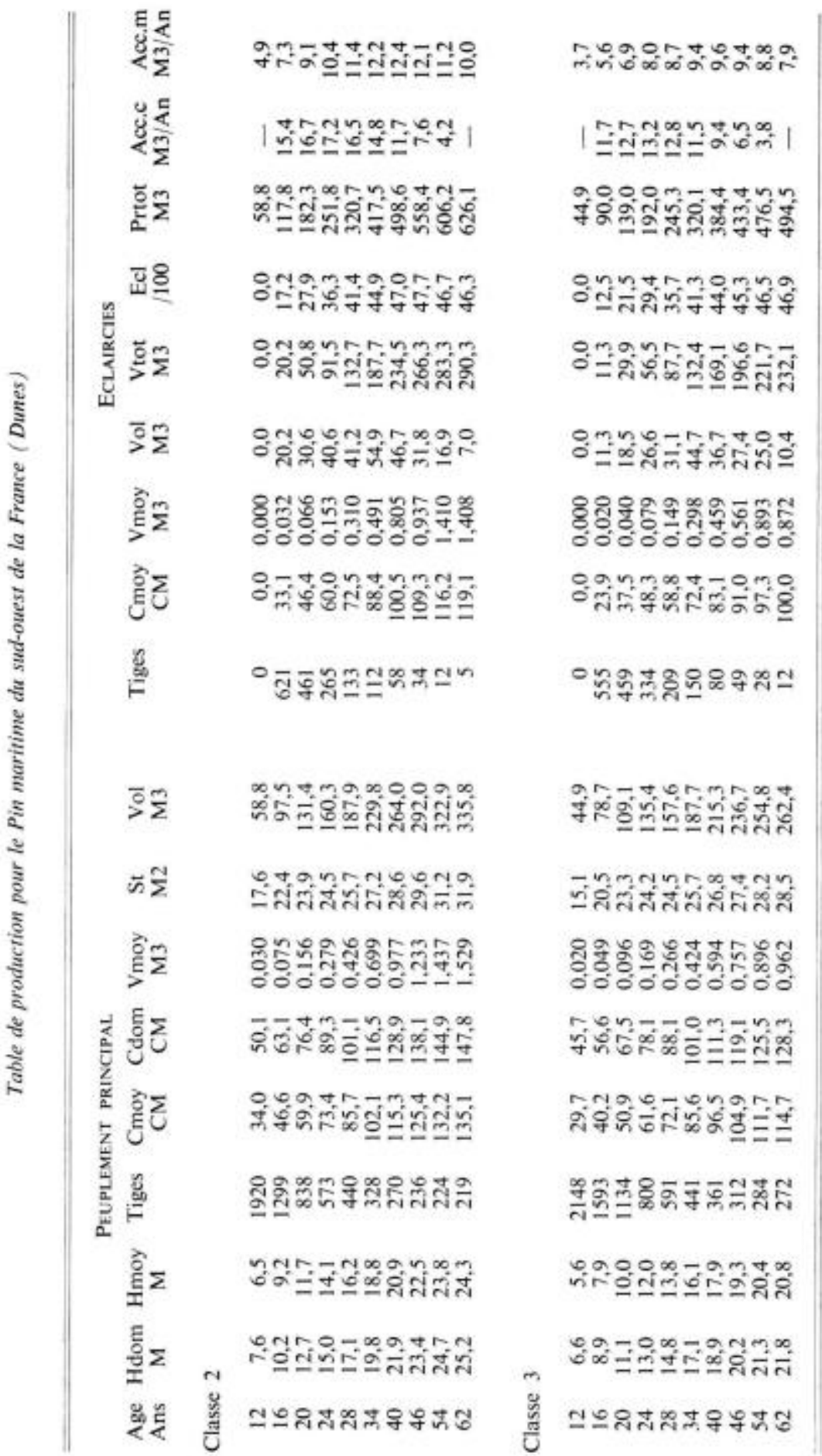


Table de production pour le Pin maritime du sud-ouest de la France (Dunss)

FEUPLFMFNT PRINCIPAL.

\begin{tabular}{|c|c|c|c|c|c|c|c|c|}
\hline Age & Hdom & Hmoy & Tiges & Cmoy & Cdom & Vmoy & St & Vol \\
\hline Ans & M & M & & $\mathrm{CM}$ & $\mathrm{CM}$ & M3 & M2 & M3 \\
\hline \multicolumn{9}{|c|}{ Classe 4} \\
\hline 12 & 5.7 & 4,6 & 2309 & 25,7 & 41,4 & 0,013 & 12.1 & 31,9 \\
\hline 16 & 7,6 & 6.5 & 1914 & 34,1 & 50,3 & 0,030 & 17,7 & 59,2 \\
\hline 20 & 9,4 & 8.3 & 1473 & 42,6 & 59,1 & 0,058 & 21,2 & 86,1 \\
\hline 24 & 11.1 & 10,0 & 1135 & 50,8 & 67,4 & 0,096 & 23,3 & 107,1 \\
\hline 28 & 12,5 & 11.5 & 859 & 59,0 & 75,4 & 0,150 & 23,8 & 129,2 \\
\hline 34 & 14,4 & 13,4 & 638 & 69,6 & 85,8 & 0.239 & 24,6 & 152,7 \\
\hline 40 & 15,9 & 14,9 & 517 & 78,4 & 94,1 & 0,332 & 25.2 & 172,0 \\
\hline 46 & 17,0 & 16.1 & 447 & 85.0 & 100,4 & 0,416 & 25,7 & 186.5 \\
\hline 54 & 17,9 & 17.0 & 397 & 90,7 & 105,8 & 0,501 & 26,0 & 199.6 \\
\hline & & & 383 & 93,0 & 107.9 & 0.536 & 26,4 & 205.8 \\
\hline
\end{tabular}

Classe 5

\begin{tabular}{|c|c|c|c|c|c|c|c|c|c|c|c|c|c|c|c|c|}
\hline 4,8 & 3,7 & 2300 & 21.9 & 37,2 & 0,009 & 8,8 & 20,8 & 0 & 0.0 & 0,000 & 0,0 & 0,0 & 0,0 & 20.8 & - & 1,7 \\
\hline 6,3 & 5,2 & 2234 & 28,2 & 44,0 & 0,017 & 14,1 & 39,6 & 66 & 2,2 & 0,015 & 1.0 & 1,0 & 2,5 & 40,7 & 5.8 & 2,5 \\
\hline 7,7 & 6,7 & 1881 & 34,7 & 50,9 & 0,032 & 18.0 & 61,2 & 353 & 14.1 & 0,016 & 5,6 & 6,7 & 9.8 & 67,2 & 6,5 & 3,3 \\
\hline 9,1 & 8,0 & 1555 & 40,9 & 57,4 & 0,052 & 20,7 & 81,0 & 326 & 25,2 & 0,016 & 5,2 & 11,9 & 12.8 & 93,0 & 6,3 & 3,8 \\
\hline 10,2 & 9,2 & 1291 & 46,8 & 63,3 & 0,076 & 22.5 & 98,1 & 264 & 33,4 & 0,033 & 8,8 & 20,7 & 17,4 & 118,9 & 6,3 & 4,2 \\
\hline 11,8 & 10,7 & 996 & 54,6 & 71,2 & 0,119 & 23.7 & 118.8 & 295 & 41,2 & 0,058 & 17,1 & 37,9 & 24,1 & 156,8 & 5,8 & 4,6 \\
\hline 13,0 & 11,9 & 810 & 61,2 & 77,6 & 0,165 & 24,1 & 134,3 & 186 & 47,8 & 0,091 & 17,0 & 55,0 & 27,0 & 189,3 & 4,8 & 4.7 \\
\hline 13,8 & 12,8 & 708 & 66,0 & 82.4 & 0.204 & 24,6 & 145,2 & 102 & 52,9 & 0,149 & 15,2 & 70,2 & 32,6 & 215,5 & 3,4 & 4,6 \\
\hline 14,6 & 13,6 & 623 & 70,4 & 86,5 & 0,247 & 24,6 & 154,3 & 85 & 57,2 & 0,149 & 12,7 & 83.0 & 34,9 & 237,3 & 1,9 & 4.3 \\
\hline 14,9 & 13,9 & 588 & 72,3 & 88,3 & 0,268 & 24,5 & 158,0 & 35 & 59,0 & 0.151 & 5,2 & $\$ 8,3$ & 35.8 & 246,3 & - & 3.9 \\
\hline
\end{tabular}

\section{ECLAIRCIFS}

$\begin{array}{cccccccc}\text { Tiges Cmoy } & \text { Vmoy } & \text { Vol } & \text { Vtol } & \text { Ecl } & \text { Prtot } & \text { Acc.c } & \text { Acc.m } \\ \text { CM } & \text { M3 } & \text { M3 } & \text { M3 } & 100 & \text { M3 } & \text { M3/An } & \text { M3/An }\end{array}$

$\begin{array}{rrrrrrrrr}0 & 0,0 & 0,000 & 0,0 & 0,0 & 0,0 & 31,9 & - & 2,6 \\ 386 & 13,0 & 0,016 & 6,1 & 6,1 & 9,4 & 65,4 & 8,4 & 4,0 \\ 441 & 28,0 & 0,016 & 7,0 & 13,2 & 13,3 & 99,4 & 9,1 & 4,9 \\ 338 & 37,5 & 0,049 & 16,5 & 29,8 & 21,4 & 138,9 & 9,7 & 5,7 \\ 276 & 45,3 & 0,065 & 17,9 & 47,8 & 27,0 & 177,0 & 9,4 & 6,3 \\ 221 & 56,5 & 0,149 & 33,0 & 80,8 & 34,6 & 233,6 & 8,6 & 6,8 \\ 121 & 65,3 & 0,233 & 28,1 & 109,0 & 38,7 & 281,0 & 6.9 & 7,0 \\ 70 & 71,9 & 0,309 & 21,6 & 130,6 & 41,1 & 317,2 & 4,8 & 6,8 \\ 50 & 77,3 & 0,368 & 18,4 & 149,1 & 42,7 & 348,7 & 2.8 & 6,4 \\ 14 & 79,6 & 0,568 & 7,9 & 157,0 & 43,2 & 362,9 & - & 5,8\end{array}$

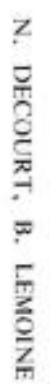


ANNEXE 2

Définition des variables

\begin{tabular}{|c|c|c|c|}
\hline $\begin{array}{l}\text { Sym- } \\
\text { bole } \\
\text { Symbol } \\
\text { Symbol }\end{array}$ & Définition & Begriff & Definition \\
\hline$A$ & Age (Années) & Alter (Jahre) & Age (years) \\
\hline$N$ & Nombre de tiges a l'hectare & Stammzahl pro ha & Number of stems per ha \\
\hline$C_{i}$ & $\begin{array}{l}\text { Circonférence à } 1,30 \mathrm{~m} \text { de } \\
\text { l'arbre de surface terrière } \\
\text { movenne }(\mathrm{cm})\end{array}$ & $\begin{array}{l}\text { Umfang in } 1,3 \mathrm{~m} \text { Höhe des } \\
\text { Grundflïchenmittelstammes } \\
(\mathrm{cm})\end{array}$ & $\begin{array}{l}\text { Circonference at } 1,3 \mathrm{~m} \text { cor- } \\
\text { responding to mean basal } \\
\text { area of stand (cm) }\end{array}$ \\
\hline$H_{i}$ & $\begin{array}{l}\text { Hauteur de l'arbre de surface } \\
\text { terriere moyenne }(\mathrm{m})\end{array}$ & $\begin{array}{l}\text { Höhe des Grundflächenmit- } \\
\text { telstammes }(\mathrm{m})\end{array}$ & $\begin{array}{l}\text { Height corresponding to mean } \\
\text { basal area }(\mathrm{m})\end{array}$ \\
\hline$V_{G}$ & $\begin{array}{l}\text { Volume de larbore de surface } \\
\text { terrière moyenne }\left(\mathrm{dm}^{3}\right)\end{array}$ & $\begin{array}{l}\text { Derbholzmasse des Grund- } \\
\text { flächenmittelstammes }\left(\mathrm{dm}^{3}\right)\end{array}$ & $\begin{array}{l}\text { Volume of tree correspond- } \\
\text { ing to mcan basal area }\left(\mathrm{dm}^{3}\right)\end{array}$ \\
\hline$H_{\circ}$ & $\begin{array}{l}\text { Hauteur de l'arbre de surface } \\
\text { terrière moyenne des } 100 \\
\text { plus gros arbres a l'hectare } \\
\text { (m) }\end{array}$ & $\begin{array}{l}\text { Höhe des Grundflächenmit- } \\
\text { telstammes der } 100 \text { stärksten } \\
\text { Bäume/ha (m) }\end{array}$ & $\begin{array}{l}\text { Height corresponding to } \\
\text { mean basal area of the } 100 \\
\text { largest trees/ha (m) }\end{array}$ \\
\hline$G$ & $\begin{array}{l}\text { Surface terrière }\left(\mathrm{m}^{2} / \mathrm{ha}\right) \mathrm{du} \\
\text { peuplement }\end{array}$ & $\begin{array}{l}\text { Grundfläche }\left(\mathrm{m}^{2} / \mathrm{ha}\right) \text { des } \\
\text { Bestandes }\end{array}$ & Basal area of stand $\left(\mathrm{m}^{2} / \mathrm{ha}\right)$ \\
\hline$V$ & $\begin{array}{l}\text { Volume du peuplement }\left(\mathrm{m}^{3}\right) \\
\text { ha) }\end{array}$ & $\begin{array}{l}\text { Derbholzmasse des Bestan- } \\
\text { des }\left(m^{3} / h a\right)\end{array}$ & Stand volume $\left(\mathrm{m}^{\mathrm{i} / \mathrm{ha}}\right)$ \\
\hline$C_{g}$ & $\begin{array}{l}\text { Circonférence de l'arbre de } \\
\text { surface terrière moyenne des } \\
\text { produits d'ćclaircie (cm) } \\
\text { Volume moyen des produits } \\
\text { d'éclaircie (dm") }\end{array}$ & $\begin{array}{l}\text { Umfang des Grundflächen- } \\
\text { mittelstammes des ausscheid- } \\
\text { enden Bestandes }(\mathrm{cm}) \\
\text { Derbholzmasse des Mittel- } \\
\text { stammes des ausscheidenden } \\
\text { Bestandes (dm:3) }\end{array}$ & $\begin{array}{l}\text { Circonference correspond- } \\
\text { ing to mean basal area of } \\
\text { thinning products (cm) } \\
\text { Mean volume of thinning } \\
\text { products }\left(\mathrm{dm}^{3}\right)\end{array}$ \\
\hline 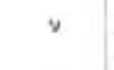 & Volume de l'éclaircie $\left(\mathrm{m}^{3} / \mathrm{ha}\right)$ & $\begin{array}{l}\text { Derbholzmasse des ausschei- } \\
\left.\text { denden Bestandes ( } \mathrm{m}^{3} / \mathrm{ha}\right)\end{array}$ & $\begin{array}{l}\text { Volume of the thinning } \\
\left(\mathrm{m}^{3} / \mathrm{ha}\right)\end{array}$ \\
\hline$g$ & $\begin{array}{l}\text { Surface terrière de l'éclaircie } \\
\left(\mathrm{m}^{2} / \mathrm{ha}\right)\end{array}$ & $\begin{array}{l}\text { Kreisfäche des ausscheiden- } \\
\text { den Bestandes }\left(\mathrm{m}^{2} / \mathrm{ha}\right)\end{array}$ & $\begin{array}{l}\text { Basal area of the thinning } \\
\left(\mathrm{m}^{2} / \mathrm{ha}\right)\end{array}$ \\
\hline$n$ & $\begin{array}{l}\text { Nombre de tiges enlevées en } \\
\text { eclaircie }\end{array}$ & $\begin{array}{l}\text { Anzahl der Stämme des aus- } \\
\text { scheidenden Bestandes }\end{array}$ & $\begin{array}{l}\text { Number of stems of the thin- } \\
\text { ning per ha }\end{array}$ \\
\hline$\Sigma_{v}$ & $\begin{array}{l}\text { Somme cumulée des volumes } \\
\text { enlevés en éclaircie }\left(\mathrm{m}^{2} / \mathrm{ha}\right)\end{array}$ & $\begin{array}{l}\text { Summe der Vorerträge an } \\
\text { Derbholz }\left(\mathrm{m}^{2} / \mathrm{ha}\right)\end{array}$ & $\begin{array}{l}\text { Total volume of the thinning } \\
\text { products }\left(\mathrm{m}^{2} / \mathrm{ha}\right)\end{array}$ \\
\hline Et & $\begin{array}{l}\text { Volume total produit depuis } \\
\text { l'origine }\left(\mathrm{m}^{2} / \mathrm{ha}\right)\end{array}$ & $\begin{array}{l}\text { Gesamtleistung an Derbholz- } \\
\text { masse }\left(\mathrm{m}^{3} / \mathrm{ha}\right)\end{array}$ & $\begin{array}{l}\text { Total crop Yield to date } \\
\left(\mathrm{m}^{3} / \mathrm{ha}\right)\end{array}$ \\
\hline$I_{e}$ & $\begin{array}{l}\text { Accroissement courant an- } \\
\text { nuel en volume ( } \mathrm{m}^{3} \text { /ha/an) }\end{array}$ & $\begin{array}{l}\text { Laufender jährlicher Zu- } \\
\text { wachs }\left(\mathrm{m}^{3} / \mathrm{ha} / \text { Jahre) }\right.\end{array}$ & $\begin{array}{l}\text { Current annual volume incre- } \\
\text { ment }\left(m^{3} / \text { ha/year }\right)\end{array}$ \\
\hline$I_{w}$ & $\begin{array}{l}\text { Accroissement moyen annuel } \\
\text { en volume depuis l'origine } \\
\left(\mathrm{m}^{3} / \mathrm{ha} / \mathrm{an}\right)\end{array}$ & $\begin{array}{l}\text { Alters-Durchnittszuwachs } \\
\left(\mathrm{m}^{3} / \mathrm{ha} / \mathrm{Jahre}\right)\end{array}$ & $\begin{array}{l}\text { Mean annual volume incre- } \\
\text { ment }\left(\mathrm{m}^{3} / \mathrm{ha} / \text { year }\right)\end{array}$ \\
\hline
\end{tabular}


ANNEXE 3

ABAQUE POUR LE CUBAGE DES PEUPLEMENTS DE LANDE

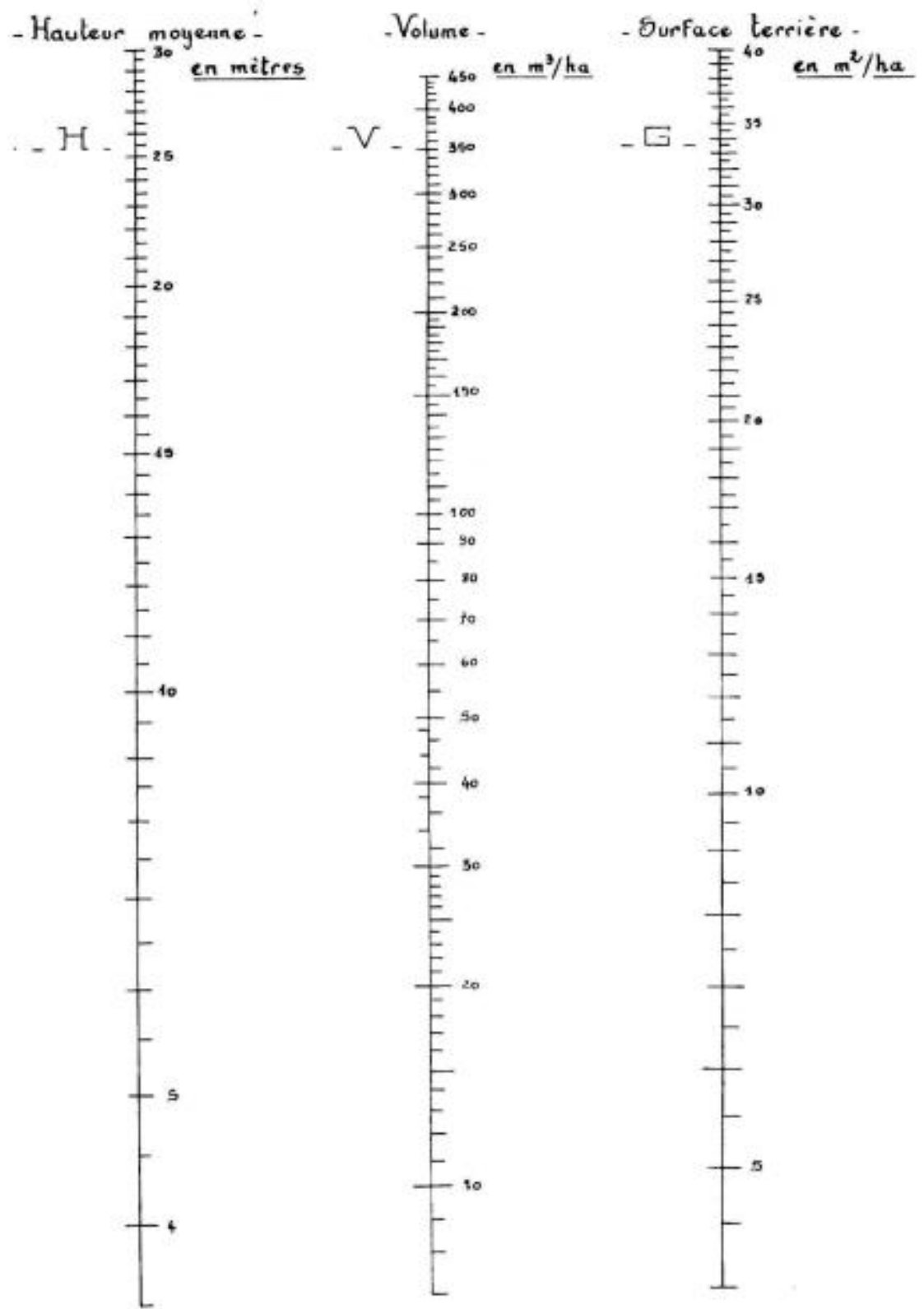


ABAQUE POUR LE CUBAGE DES PEUPLEMENTS DE DUNE

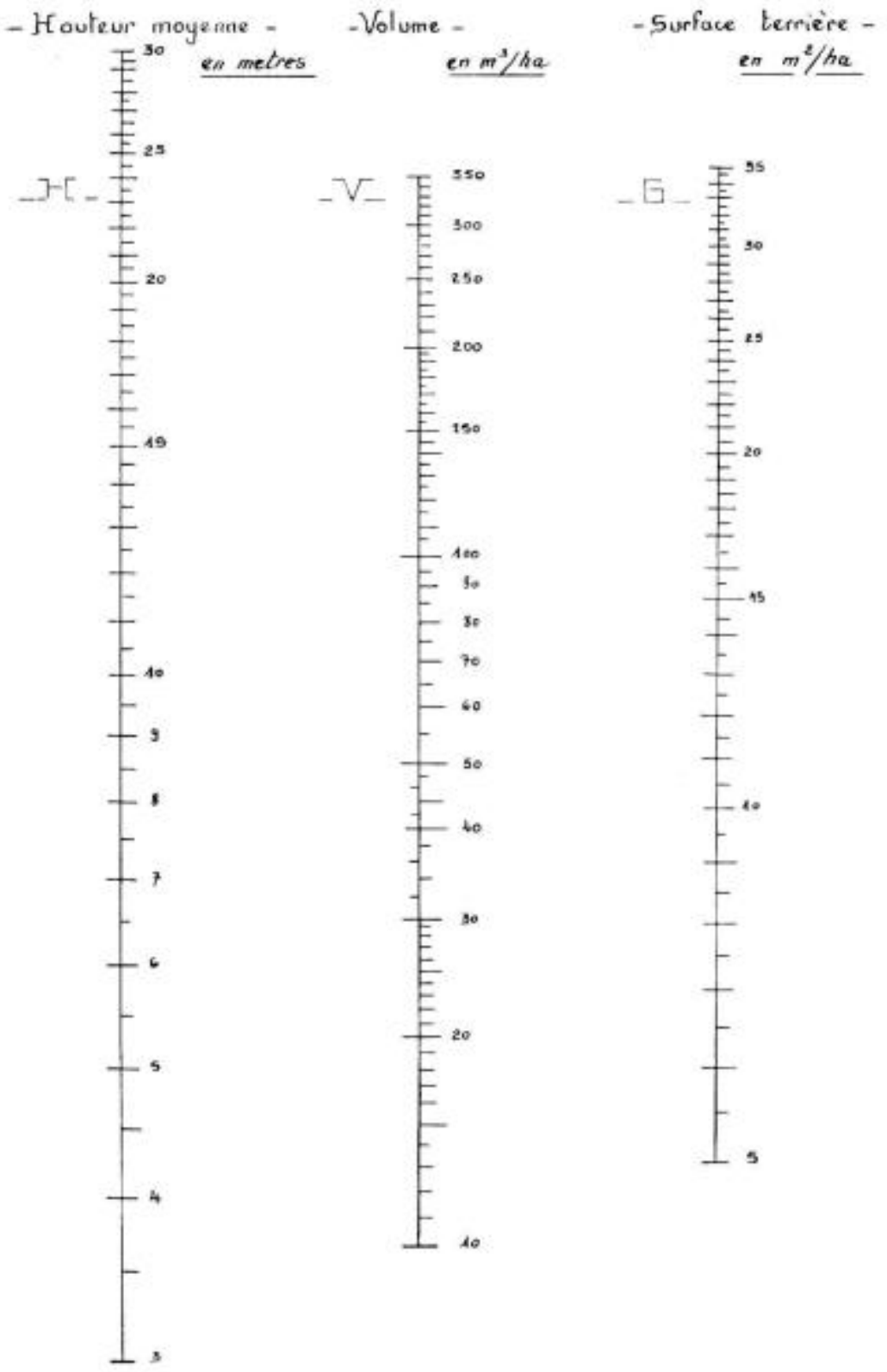


ANNEXE 4

Correspondance entre $C_{1,30,} C_{3+10}$ et $C_{4+0}$

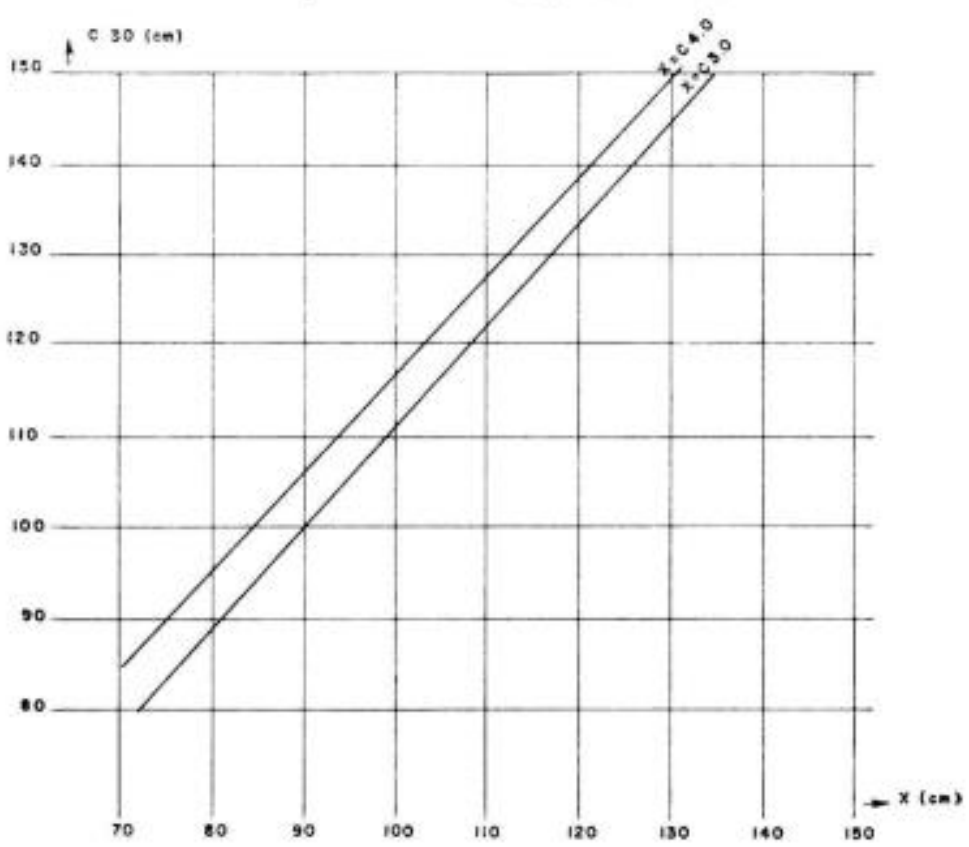

